\title{
Nonlinear resonant frequency of graphene/elastic/piezoelectric laminated films under active electric loading
}

\author{
H.B. Li, F.P. Yang and X Wang ${ }^{*}$ \\ School of Naval Architecture, Ocean and Civil Engineering \\ (State Key Laboratory of Ocean Engineering), \\ Shanghai Jiaotong University, Shanghai 200240, People's Republic China.
}

\begin{abstract}
This paper reports a result of investigation on the nonlinear resonant characteristics of graphene/elastic/piezoelectric (GEP) asymmetric laminated films under exciting electric loading, based on nonlocal elastic theory and von Karman strain-displacement relations in an active control system. The nonlinear dynamic governing equations containing deflection function, stress function and electric potential function are firstly constructed by Hamilton's principle, then are solved by the iterative homotopy harmonic balance method. Results show that the nonlinear resonant frequency of GEP laminated films can be controlled by adjusting the voltage exerted on piezoelectric layer according to engineering requirement, and the material property of elastic layer has a significant effect on the nonlinear resonant frequency of GEP laminated films, where the nonlinear behavior of resonant frequencies increases as the ratio of vibration amplitude to thickness of laminated films increases.
\end{abstract}

Keywords: Layered structures, Thin films, Nonlinear vibration, Analytical modeling

\footnotetext{
* Corresponding author. Email address: xwang@sjtu.edu.cn; Tel.and fax: 086-021-54745367.
} 


\section{Introduction}

Graphene sheet and graphene-related materials have been attracting tremendous interest since it was firstly exfoliated and reported [1] owing to its superior mechanical, electrical, thermal and optical properties [2-4], as well as the characteristic of ultrahigh frequency range up to the terahertz order [5]. These distinguished properties have made graphene sheet be worldwide attention related to the use of nanosensors, nanoresonators, nanoelectronics, batteries, super-capacitors, solar cells and so on $[6,7]$. On the other hand, due to the unique electro-mechanical conversion capability, piezoelectric material as a smart material is widely used in monitoring and controlling the dynamic characteristics of micro-electromechanical systems (MEMS) or nano-electromehcanical systems (NEMS) [8]. However Graphene is non-piezoelectric, which restrains its applications. Though recent discovery revealed that graphene could be coaxed into a piezoelectric material [9], it still stays in the phase of quantum mechanical calculations. Thus it is envisaged that why not coupling these two materials to make fully use of their distinctive material properties.

Luckily, the laminated films composed of grapheme and piezoelectric $(\mathrm{ZnO}$, PVDF, PZT, LiNbO3, etc) are successfully synthesized because of some emerging technology such as inkjet printing [10], spin coating [11], vacuum filtration [12], and the Langmuir-Blodgett film technique [13], etc. At macro scale, for example, Shin et al. [10] and Xu et al. [14] fabricated a kind of Graphene-based loud speaker which contains a layer polyvinylidene fluoride (PVDF) and two layers graphene at the top and bottom. Lee et al. [11] reported a new type of fully flexible transparent piezoelectric nanogenerator consisting of poly and graphene electrodes [15]. At micro or nano scale, Zeng et al. [16] studied the mechanism of mechanically stable $\mathrm{ZnO}$ /graphene films with excellent optical properties using pulsed laser deposited technology. Jie et al. [17] transferred CVD synthetic monolayer graphene sheet to PMN-PT piezoelectric substrates and studied the effect of tunable strain on the Raman spectra via applying the electric field at the deposited 150nm-thick Au electrodes. In 
another work, by coating LiNbO3 on graphene, a novel scheme was demonstrated by Battista [18] to improve the absorption of solar radiation in a solar energy harvesting system. In view of these graphene/piezoelectric related experimental works, it is predictably that graphene/piezoelectric composites based electrical elements will soon be applied in energy harvesting, disease detection, constant monitoring of material states and other fields.

Generally, resonant frequency is one of the core parameters in the dynamic design of micro or nano-devises, so it is helpful to have a good understanding on the resonant frequencies of sensing systems.

In early reports, the vibration characteristics of laminated piezoelectric structures have been described based different methods [19-29]. However, most of these investigations are focused on the linear vibration characteristics, and no considering the nonlocal effect which has a significant effect on the physical properties of nano-laminated structures.

So far, some experimental, numerical and analytical methods are utilized to investigate the mechanical and physical properties of nano-structures [30-32]. By utilizing an experimental method [30], the physical behaviors of multi-walled carbon nanotubes are described. But it is difficult to control experiments at nanoscale, so it is nearly impossible to directly measure their properties. The dynamic behaviors of nanostructures can be simulated [31, 32] based on the molecular dynamics (MD) method, but the computational problem is that the larger time history involved in the MD simulations. In past years, a nonlocal continuum theory containing the long range force among atoms is widely used to study the influence of an internal scale effect on the physical behaviors of nano-structures [33-37]. For example, Lu et al. [38] had an elaborate investigation on two kinds of nonlocal elastic plate theories, and the effect of nonlocal parameter on the natural frequencies of symmetrically orthotropic plates was discussed. In another literature, Nazemnezhad and Hosseini-Hashemi [39] investigated the natural frequencies of cantilever multi-layer graphene nanoribbons by comparing the results obtained by the MD method and nonlocal elasticity theory, respectively. 
The primary object of this paper is to investigate the nonlinear resonant frequency of graphene/elastic/piezoelectric (GEP) asymmetric laminated films based on a nonlocal continuum theory containing the long range force among atoms and von Karman nonlinear geometric relation in an active control system. The nonlinear dynamic governing equations containing deflection function, stress function and electric potential function are firstly found utilizing Hamilton's principle, then are solved using the iterative homotopy harmonic balance method. In the numerical examples presented, the effects of some impact factors, including material properties, scale effect, geometric shape and ratio of side length to thickness, vibration amplitude and mode, and active voltage are considered. Results show that the applied positive voltage decreases the nonlinear resonant frequency of GEP laminated films, and the applied negative voltage induces the nonlinear resonant frequency to increase; the nonlinear behavior of resonant frequencies nonlinearly increases as the ratio of vibration amplitude to thickness increases, and appears the maximum value for the elastic layer made of metal $\mathrm{W}$ owing to its larger elastic coefficients; the nonlinear resonant frequency predicted by nonlocal theory is lower than that from classical continuation theory, and the nonlinear resonant frequency decreases as the small scale effect increases when the non-dimensional ratio of vibration amplitude to thickness is larger than a given value. Some meaningful results in this paper will stimulate further interest in this topic and may be used as a useful reference for the application and the design of various nano-devices as high frequency antenna, signal generator, batteries, super-capacitors, solar cells etc. in which graphene sheet laminated films act as basic elements.

\section{Nonlinear governing equation of GEP films considering small scale effect}

The schematic of a GEP (graphene/elastic/piezoelectric) asymmetric laminated films with a piezoelectric control system is shown in Fig. 1, where the plane size and thickness of the GEP films are, respectively, given by dimension $a \times b$ (for square film, $\left.L_{x}=L_{y}=a=b\right)$ and $h$.

Due to the transverse deflection of GEP laminated films is much larger than the 
in-plane displacements of the GEP laminated films, the in-plane displacements is negligible so that the stress-strain relations of piezoelectric material based on nonlocal elastic theory are written as

$$
\begin{aligned}
& \left(1-\mu^{2} \nabla^{2}\right)\left\{\begin{array}{l}
\sigma_{x} \\
\sigma_{y} \\
\sigma_{x y}
\end{array}\right\}=\left[\begin{array}{ccc}
C_{11}^{p} & C_{12}^{p} & 0 \\
C_{12}^{p} & C_{22}^{p} & 0 \\
0 & 0 & C_{66}^{p}
\end{array}\right]\left\{\begin{array}{l}
\varepsilon_{x} \\
\varepsilon_{y} \\
\gamma_{x y}
\end{array}\right\}-\left[\begin{array}{ccc}
0 & 0 & e_{31} \\
0 & 0 & e_{32} \\
0 & 0 & 0
\end{array}\right]\left\{\begin{array}{l}
E_{x} \\
\left.E_{y}\right\} \\
E_{z}
\end{array}\right\} \\
& \left(1-\mu^{2} \nabla^{2}\right)\left\{\begin{array}{l}
D_{x} \\
D_{y} \\
D_{z}
\end{array}\right\}=\left[\begin{array}{ccc}
0 & 0 & 0 \\
0 & 0 & 0 \\
e_{31} & e_{32} & 0
\end{array}\right]\left\{\begin{array}{l}
\varepsilon_{x} \\
\varepsilon_{y} \\
\gamma_{x y}
\end{array}\right\}+\left[\begin{array}{ccc}
g_{11} & 0 & 0 \\
0 & g_{22} & 0 \\
0 & 0 & g_{33}
\end{array}\right]\left\{\begin{array}{l}
E_{x} \\
E_{y} \\
E_{z}
\end{array}\right\}
\end{aligned}
$$

where $\mu=e_{0} l$ represents the small scale parameter containing the long-range force between atoms, in which $e_{0}$ is a constant appropriate of materials and $l$ is the internal characteristic length of material depending on distance between two bonds as atom diameters; $C_{i j}^{p}, g_{i j}, e_{i j}$ are elastic constants, dielectric constants and piezoelectric constants of piezoelectric layer material, respectively; $\sigma_{i j}, D_{i}$ express the components of nonlocal stress and electric displacement, respectively; $\varepsilon_{i j}, E_{i}$ are the strain tensor and electric field vector, respectively. The relations between electric fields $E_{x, y, z}$ and electric potential $\Phi$ in a Cartesian coordinates are expressed as

$$
E_{x}=-\frac{\partial \Phi}{\partial x}, E_{y}=-\frac{\partial \Phi}{\partial y}, E_{z}=-\frac{\partial \Phi}{\partial z}
$$

As an active control system, an external control voltage $V$ is applied across the thickness of piezoelectric layer in GEP laminated films, which induces the electric potential distribution along the thickness of the piezoelectric layer. The electric potential distribution satisfying Maxwell equation may be described by a cosine variation combining with linear variation across the thickness of piezoelectric layer [40], as follows

$$
\Phi(x, y, z, t)=-\cos \left(\frac{\pi\left(z+\left(h_{2}+h_{3}\right) / 2\right)}{h_{1}}\right) \phi(x, y, t)+\frac{2\left(z+\left(h_{2}+h_{3}\right) / 2\right) V}{h_{1}} e^{i \omega t}
$$

where $\quad z \in\left[-\left(h_{1}+h_{2}+h_{3}\right) / 2,\left(h_{1}-h_{2}-h_{3}\right) / 2\right]$ denotes the electric potential 
distribution through the thickness of piezoelectric layer; $\phi(x, y, t)$ is the electric potential in the middle surface of piezoelectric layer; $\omega$ is the natural frequency of GEP films. And the coordinate of a rectangular GEP laminated films in the $z$ direction is shown in Fig. 2. Substituting Eq. (5) into Eq. (4), the components of electric field strength are expressed as

$$
\begin{gathered}
E_{x}=\cos \left(\frac{\pi\left(z+\left(h_{2}+h_{3}\right) / 2\right)}{h_{1}}\right) \frac{\partial \phi}{\partial x}, E_{y}=\cos \left(\frac{\pi\left(z+\left(h_{2}+h_{3}\right) / 2\right)}{h_{1}}\right) \frac{\partial \phi}{\partial y}, \\
E_{z}=-\frac{\pi}{h_{1}} \sin \left(\frac{\pi\left(z+\left(h_{2}+h_{3}\right) / 2\right)}{h_{1}}\right) \phi-\frac{2 V}{h_{1}} e^{i \omega t}
\end{gathered}
$$

In addition, the nonlocal stress-strain relationship for graphene layer and elastic layer can be written, respectively, as

$$
\left(1-\mu^{2} \nabla^{2}\right)\left\{\begin{array}{l}
\sigma_{x x} \\
\sigma_{y y} \\
\sigma_{x y}
\end{array}\right\}=\left[\begin{array}{ccc}
C_{11}^{i} & C_{12}^{i} & 0 \\
C_{12}^{i} & C_{22}^{i} & 0 \\
0 & 0 & C_{66}^{i}
\end{array}\right]\left\{\begin{array}{l}
\varepsilon_{x x} \\
\varepsilon_{y y} \\
\gamma_{x y}
\end{array}\right\}
$$

where the superscript $i=g$ or $e$ represent the components of elastic coefficients of graphene layer or elastic layer, respectively.

The nonlocal force and moment resultants of GEP laminated films are written as

$$
\left\{\begin{array}{l}
N_{x} \\
N_{y} \\
N_{x y}
\end{array}\right\}=\sum_{k=1}^{N} \int_{z_{k-1}}^{z_{k}}\left\{\begin{array}{l}
\sigma_{x} \\
\sigma_{y} \\
\sigma_{x y}
\end{array}\right\} d z,\left\{\begin{array}{l}
M_{x} \\
M_{y} \\
M_{x y}
\end{array}\right\}=\sum_{k=1}^{N} \int_{z_{k-1}}^{z_{k}}\left\{\begin{array}{l}
\sigma_{x} \\
\sigma_{y} \\
\sigma_{x y}
\end{array}\right\} z d z
$$

Substituting Eqs. (1)-(6) into Eq. (7), gives

$$
\begin{aligned}
\left(1-\mu^{2} \nabla^{2}\right)\left[\begin{array}{c}
N_{x} \\
N_{y} \\
N_{x y}
\end{array}\right] & =\left(1-\mu^{2} \nabla^{2}\right)\left[\begin{array}{c}
N_{x}^{0} \\
N_{y}^{0} \\
N_{x y}^{0}
\end{array}\right]+2 V\left[\begin{array}{c}
e_{31} \\
e_{32} \\
0
\end{array}\right] \\
& =\left[\begin{array}{ccc}
A_{11} & A_{12} & 0 \\
A_{12} & A_{22} & 0 \\
0 & 0 & A_{66}
\end{array}\right]\left[\begin{array}{c}
\varepsilon_{x}^{0} \\
\varepsilon_{y}^{0} \\
\gamma_{x y}^{0}
\end{array}\right]+\left[\begin{array}{ccc}
B_{11} & B_{12} & 0 \\
B_{12} & B_{22} & 0 \\
0 & 0 & B_{66}
\end{array}\right]\left[\begin{array}{c}
K_{x} \\
K_{y} \\
K_{x y}
\end{array}\right]+2 V\left[\begin{array}{c}
e_{31} \\
e_{32} \\
0
\end{array}\right]
\end{aligned}
$$




$$
\begin{aligned}
\left(1-\mu^{2} \nabla^{2}\right)\left[\begin{array}{c}
M_{x} \\
M_{y} \\
M_{x y}
\end{array}\right] & =\left[\begin{array}{ccc}
B_{11} & B_{12} & 0 \\
B_{12} & B_{22} & 0 \\
0 & 0 & B_{66}
\end{array}\right]\left[\begin{array}{c}
\varepsilon_{x}^{0} \\
\varepsilon_{y}^{0} \\
\gamma_{x y}^{0}
\end{array}\right] \\
& +\left[\begin{array}{ccc}
D_{11} & D_{12} & 0 \\
D_{12} & D_{22} & 0 \\
0 & 0 & D_{66}
\end{array}\right]\left[\begin{array}{c}
K_{x} \\
K_{y} \\
K_{x y}
\end{array}\right]+\left(\frac{2}{\pi} h_{1} \phi+\Lambda\right)\left[\begin{array}{c}
e_{31} \\
e_{32} \\
0
\end{array}\right]
\end{aligned}
$$

where $A_{i j}=\sum_{k=1}^{N}\left[C_{i j}\right]_{k}\left(z_{k}-z_{k-1}\right), B_{i j}=\frac{1}{2} \sum_{k=1}^{N}\left[C_{i j}\right]_{k}\left(z_{k}^{2}-z_{k-1}^{2}\right), D_{i j}=\frac{1}{3} \sum_{k=1}^{N}\left[C_{i j}\right]_{k}\left(z_{k}^{3}-z_{k-1}^{3}\right)$

represent the stretch stiffness matrix, coupling stiffness matrix and bending stiffness matrix of GEP laminated films, respectively; $\Lambda=\left(h_{2}+h_{3}\right) V e^{i \omega t}$ is a time function.

The large amplitude motion of GEP films is induced by the von Karman nonlinear geometric relation, as follows

$$
\begin{aligned}
& \varepsilon_{x}=\frac{\partial u}{\partial x}+\frac{1}{2}\left(\frac{\partial w}{\partial x}\right)^{2}-z \frac{\partial^{2} w}{\partial x^{2}}=\varepsilon_{x}^{0}+z K_{x} \\
& \varepsilon_{y y}=\frac{\partial v}{\partial y}+\frac{1}{2}\left(\frac{\partial w}{\partial y}\right)^{2}-z \frac{\partial^{2} w}{\partial y^{2}}=\varepsilon_{y}^{0}+z K_{y} \\
& \gamma_{x y}=\frac{\partial u}{\partial y}+\frac{\partial v}{\partial x}+\frac{\partial w}{\partial x} \frac{\partial w}{\partial y}-2 z \frac{\partial^{2} w}{\partial x \partial y}=\varepsilon_{x y}^{0}+z K_{x y}
\end{aligned}
$$

where $K_{x}=-\frac{\partial^{2} w}{\partial x^{2}}, K_{y}=-\frac{\partial^{2} w}{\partial y^{2}}$ and $K_{x y}=-2 \frac{\partial^{2} w}{\partial x \partial y} \cdot u, v, w$ are the displacements of GEP films in the $x, y$ and $z$ directions, respectively.

Utilizing the Hamilton's principle [41], the nonlinear dynamic governing equations for GEP films with an active control system are given by

$$
\begin{aligned}
& \delta u: \frac{\partial N_{x}}{\partial x}+\frac{\partial N_{x y}}{\partial y}=I_{0} \frac{\partial^{2} u}{\partial t^{2}}-I_{1} \frac{\partial^{3} w}{\partial x \partial t^{2}} \\
& \delta v: \frac{\partial N_{x y}}{\partial x}+\frac{\partial N_{y}}{\partial y}=I_{0} \frac{\partial^{2} v}{\partial t^{2}}-I_{1} \frac{\partial^{3} w}{\partial y \partial t^{2}}
\end{aligned}
$$




$$
\begin{gathered}
\delta w: \frac{\partial^{2} M_{x}}{\partial x^{2}}+2 \frac{\partial^{2} M_{x y}}{\partial x \partial y}+\frac{\partial^{2} M_{y}}{\partial y^{2}}+\frac{\partial}{\partial x}\left(N_{x} \frac{\partial w}{\partial x}+N_{x y} \frac{\partial w}{\partial y}\right)+\frac{\partial}{\partial y}\left(N_{x y} \frac{\partial w}{\partial x}+N_{y} \frac{\partial w}{\partial y}\right) \\
=I_{0} \frac{\partial^{2} w}{\partial t^{2}}+I_{1}\left(\frac{\partial^{3} u}{\partial x \partial t^{2}}+\frac{\partial^{3} v}{\partial y \partial t^{2}}\right)-I_{2}\left(\frac{\partial^{4} w}{\partial x^{2} \partial t^{2}}+\frac{\partial^{4} w}{\partial y^{2} \partial t^{2}}\right) \\
\delta \phi: \int_{z_{0}}^{z_{1}}\left[\cos \left(\frac{\pi\left(z+\left(h_{2}+h_{3}\right) / 2\right)}{h_{1}}\right) \frac{\partial D_{x}}{\partial x}+\cos \left(\frac{\pi\left(z+\left(h_{2}+h_{3}\right) / 2\right)}{h_{1}}\right) \frac{\partial D_{y}}{\partial x}\right. \\
\left.+\frac{\pi}{h_{1}} \sin \left(\frac{\pi\left(z+\left(h_{2}+h_{3}\right) / 2\right)}{h_{1}}\right) D_{z}\right] d z=0
\end{gathered}
$$

where

$$
\left(I_{0}, I_{1}, I_{2}\right)=\sum_{k=1}^{n} \int_{z_{k-1}}^{z_{k}} \rho_{k}\left(1, z, z^{2}\right) d z
$$

The stress function $\Psi$ is defined as

$$
N_{x}^{0}=\frac{\partial^{2} \Psi}{\partial y^{2}}, N_{y}^{0}=\frac{\partial^{2} \Psi}{\partial x^{2}}, N_{x y}^{0}=-\frac{\partial^{2} \Psi}{\partial x \partial y}
$$

, and Eqs. (11a) and (11b) are rewritten as

$$
\frac{\partial^{2} u}{\partial t^{2}}=\frac{I_{1}}{I_{0}} \frac{\partial^{3} w}{\partial x \partial t^{2}}, \frac{\partial^{2} v}{\partial t^{2}}=\frac{I_{1}}{I_{0}} \frac{\partial^{3} w}{\partial y \partial t^{2}}
$$

Multiplying $\left[S_{i j}\right]=\left[A_{i j}\right]^{-1}$ at two sides of Eq. (8), yields

$$
\left[\begin{array}{l}
\varepsilon_{x}^{0} \\
\varepsilon_{y}^{0} \\
\gamma_{x y}^{0}
\end{array}\right]=\left(1-\mu^{2} \nabla^{2}\right)\left[S_{i j}\right]\left[\begin{array}{l}
\frac{\partial^{2} \Psi}{\partial y^{2}} \\
\frac{\partial^{2} \Psi}{\partial x^{2}} \\
-\frac{\partial^{2} \Psi}{\partial x \partial y}
\end{array}\right]+\left[R_{i j}\right]\left[\begin{array}{l}
\frac{\partial^{2} w}{\partial x^{2}} \\
\frac{\partial^{2} w}{\partial y^{2}} \\
2 \frac{\partial^{2} w}{\partial x \partial y}
\end{array}\right]
$$

where $\left[R_{i j}\right]=\left[A_{i j}\right]^{-1}\left[B_{i j}\right]$ is a newly introduced matrix. Substituting Eqs. (8), (9), (12)-(15) into Eq. (11c), the nonlinear dynamic governing equation in terms of the normal deflection function $w(x, y, t)$, stress function $\Psi(x, y, t)$ and electric potential function $\phi(x, y, t)$ is derived as 


$$
\begin{aligned}
& D_{1}^{*} \frac{\partial^{4} w}{\partial x^{4}}+D_{2}^{*} \frac{\partial^{4} w}{\partial x^{2} \partial y^{2}}+D_{3}^{*} \frac{\partial^{4} w}{\partial y^{4}}-\left(1-\mu^{2} \nabla^{2}\right)\left[D_{4}^{*} \frac{\partial^{4} \Psi}{\partial x^{4}}+D_{5}^{*} \frac{\partial^{4} \Psi}{\partial x^{2} \partial y^{2}}+D_{6}^{*} \frac{\partial^{4} \Psi}{\partial y^{4}}\right]+D_{7}^{*} \frac{\partial^{2} \phi}{\partial x^{2}}+D_{8}^{*} \frac{\partial^{2} \phi}{\partial y^{2}} \\
& +\left(1-\mu^{2} \nabla^{2}\right)\left(D_{9}^{*} \frac{\partial^{2} w}{\partial t^{2}}+D_{10}^{*}\left(\frac{\partial^{4} w}{\partial x^{2} \partial t^{2}}+\frac{\partial^{4} w}{\partial y^{2} \partial t^{2}}\right)\right)+\left(D_{11}^{*} \frac{\partial^{2} w}{\partial x^{2}}+D_{12}^{*} \frac{\partial^{2} w}{\partial y^{2}}\right) \\
& -\left(\frac{\partial^{2} w}{\partial x^{2}} \frac{\partial^{2} \Psi}{\partial y^{2}}-2 \frac{\partial^{2} w}{\partial x \partial y} \frac{\partial^{2} \Psi}{\partial x \partial y}+\frac{\partial^{2} w}{\partial y^{2}} \frac{\partial^{2} \Psi}{\partial x^{2}}\right)=0
\end{aligned}
$$

where

$$
\begin{aligned}
& D_{1}^{*}=D_{11}-\left(B_{11} R_{11}+B_{12} R_{12}\right), \\
& D_{2}^{*}=2\left(D_{12}+2 D_{66}\right)-\left(B_{11} R_{12}+B_{12} R_{22}+B_{12} R_{11}+B_{22} R_{12}+2 B_{66} R_{66}\right), \\
& D_{3}^{*}=D_{22}-\left(B_{12} R_{12}+B_{22} R_{22}\right), D_{4}^{*}=\left(B_{11} S_{12}+B_{12} S_{22}\right), \\
& D_{5}^{*}=\left(B_{11} S_{11}+2 B_{12} S_{12}+B_{22} S_{22}-B_{66} S_{66}\right), D_{6}^{*}=\left(B_{12} S_{11}+B_{22} S_{12}\right), \\
& D_{7}^{*}=-\frac{2}{\pi} h_{1} e_{31}, D_{8}^{*}=-\frac{2}{\pi} h_{1} e_{32}, D_{9}^{*}=I_{0}, D_{10}^{*}=\left(\frac{I_{1}^{2}}{I_{0}}-I_{2}\right), \\
& D_{11}^{*}=-2 V e_{31}, D_{12}^{*}=-2 V e_{32} .
\end{aligned}
$$

The electrical displacement in Eq. (2) can be rewritten as

$$
\begin{gathered}
\left(1-\mu^{2} \nabla^{2}\right) D_{x}=g_{11} E_{x}=g_{11} \cos \left(\frac{\pi\left(z+\left(h_{2}+h_{3}\right) / 2\right)}{h_{1}}\right) \frac{\partial \phi}{\partial x}, \\
\left(1-\mu^{2} \nabla^{2}\right) D_{y}=g_{22} E_{y}=g_{22} \cos \left(\frac{\pi\left(z+\left(h_{2}+h_{3}\right) / 2\right)}{h_{1}}\right) \frac{\partial \phi}{\partial y}, \\
\left(1-\mu^{2} \nabla^{2}\right) D_{z}=e_{31} \varepsilon_{x}+e_{32} \varepsilon_{y}+g_{33} E_{z}=e_{31} \varepsilon_{x}+e_{32} \varepsilon_{y}-g_{33} \frac{\pi}{h_{1}} \sin \left(\frac{\pi\left(z+\left(h_{2}+h_{3}\right) / 2\right)}{h_{1}}\right) \phi
\end{gathered}
$$

Substituting above equations into Eq. (11d), yields

$$
\frac{g_{11} h_{1}}{2} \frac{\partial^{2} \phi}{\partial x^{2}}+\frac{g_{22} h_{1}}{2} \frac{\partial^{2} \phi}{\partial y^{2}}-\frac{2 h_{1} e_{31}}{\pi} \frac{\partial^{2} w}{\partial x^{2}}-\frac{2 h_{1} e_{32}}{\pi} \frac{\partial^{2} w}{\partial y^{2}}-\frac{g_{33} h_{1}}{2}\left(\frac{\pi}{h_{1}}\right)^{2} \phi=0
$$

Eqs. (16) and (19) express two fundamental dynamic governing equations containing three undetermined functions $w(x, y, t), \Psi(x, y, t)$ and $\phi(x, y, t)$. Therefore, one additional governing equation is needed to obtain the three undetermined functions. Considering the mid-plane tension of GEP laminated films induced by large deflection deformation, the compatibility equation containing the 
in-plane strain components is given by

$$
\frac{\partial^{2} \varepsilon_{x}^{0}}{\partial y^{2}}+\frac{\partial^{2} \varepsilon_{y}^{0}}{\partial x^{2}}-\frac{\partial^{2} \gamma_{x y}^{0}}{\partial x \partial y}=\left(\frac{\partial^{2} w}{\partial x \partial y}\right)^{2}-\frac{\partial^{2} w}{\partial x^{2}} \frac{\partial^{2} w}{\partial y^{2}}
$$

The above equation is rewritten as

$$
\begin{aligned}
& \left(1-\mu^{2} \nabla^{2}\right)\left(S_{11} \frac{\partial^{4} \Psi}{\partial x^{4}}+2\left(S_{12}+2 S_{66}\right) \frac{\partial^{4} \Psi}{\partial x^{2} \partial y^{2}}+S_{22} \frac{\partial^{4} \Psi}{\partial y^{4}}\right)=-R_{12} \frac{\partial^{4} w}{\partial x^{4}} \\
& -\left(R_{11}+R_{22}-2 R_{66}\right) \frac{\partial^{4} w}{\partial x^{2} \partial y^{2}}-R_{12} \frac{\partial^{4} w}{\partial y^{4}}+\left(\frac{\partial^{2} w}{\partial x \partial y}\right)^{2}-\frac{\partial^{2} w}{\partial x^{2}} \frac{\partial^{2} w}{\partial y^{2}}
\end{aligned}
$$

Hitherto the nonlinear dynamic governing equations of GEP films have been successfully constructed by Eqs. (16), (19) and (21).

\section{Boundary conditions and solving method}

In this study, the rectangle GEP laminated films is considered as clamped support along all edges, as follows

$$
\left\{\begin{array}{l}
x=0, a: \quad w=0, \frac{\partial w}{\partial x}=0, \frac{1}{b} \int_{0}^{b} N_{x y} d y=0, \quad \int_{0}^{b} \int_{0}^{a} \frac{\partial u}{\partial x} d x d y=0 \\
y=0, b: \quad w=0, \quad \frac{\partial w}{\partial y}=0, \quad \frac{1}{a} \int_{0}^{a} N_{x y} d x=0, \quad \int_{0}^{b} \int_{0}^{a} \frac{\partial v}{\partial y} d x d y=0
\end{array}\right.
$$

To satisfy the normal deflection boundary conditions, the deflection function is written as

$$
w(x, y, t)=W(t)\left(1-\cos \left(\alpha_{m} x\right)\right)\left(1-\cos \left(\beta_{n} y\right)\right)
$$

where $\alpha_{m}=\frac{2 m \pi}{a}$ and $\beta_{n}=\frac{2 n \pi}{b} . m$ and $n$ are mode numbers in the $x, y$ directions, respectively. Substituting Eq. (23) into Eq. (19), the electric potential function is expressed as

$$
\phi(x, y, t)=\left(\varphi_{1} \cos \left(\alpha_{m} x\right) \cos \left(\beta_{n} y\right)+\varphi_{2} \cos \left(\alpha_{m} x\right)+\varphi_{3} \cos \left(\beta_{n} y\right)\right) W(t)
$$

where

$$
\varphi_{1}=-\frac{P_{1} \alpha_{m}^{2}+P_{2} \beta_{n}^{2}}{Q_{1} \alpha_{m}^{2}+Q_{2} \beta_{n}^{2}-T_{1}}, \varphi_{2}=\frac{P_{1} \alpha_{m}^{2}}{Q_{1} \alpha_{m}^{2}-T_{1}}, \varphi_{3}=\frac{P_{2} \beta_{n}^{2}}{Q_{2} \beta_{n}^{2}-T_{1}}
$$

and 


$$
P_{1}=-\frac{2 h_{1} e_{31}}{\pi}, P_{2}=-\frac{2 h_{1} e_{32}}{\pi}, Q_{1}=\frac{g_{11} h_{1}}{2}, Q_{2}=\frac{g_{22} h_{1}}{2}, T_{1}=-\frac{g_{33} h_{1}}{2}\left(\frac{\pi}{h_{1}}\right)^{2} .
$$

To satisfy the compatibility equation, deflection function and in-plane boundary conditions, the general solutions for stress function $\Psi(x, y, t)$ can be expressed as

$$
\begin{aligned}
\Psi(x, y, t)= & {\left[A_{1} \cos \left(2 \alpha_{m} x\right)+A_{2} \cos \left(2 \beta_{n} y\right)+A_{3} \cos \left(2 \alpha_{m} x\right) \cos \left(\beta_{n} y\right)+A_{4} \cos \left(\alpha_{m} x\right) \cos \left(2 \beta_{n} y\right)\right.} \\
& \left.+A_{5} \cos \left(\alpha_{m} x\right) \cos \left(\beta_{n} y\right)+A_{6} \cos \left(\alpha_{m} x\right)+A_{7} \cos \left(\beta_{n} y\right)\right] W(t)^{2} \\
& +\left[B_{1} \cos \left(\alpha_{m} x\right) \cos \left(\beta_{n} y\right)+B_{2} \cos \left(\alpha_{m} x\right)+B_{3} \cos \left(\beta_{n} y\right)\right] W(t) \\
& +\left(\frac{1}{2} C_{1} x^{2}+\frac{1}{2} C_{2} y^{2}-C_{3} x y\right) W(t)^{2}
\end{aligned}
$$

Substituting Eq. (25) into Eq. (21), comparing the coefficients of same items, and utilizing the in-plane boundary conditions in Eq. (22), the undetermined constants in Eq. (25) are easily obtained as shown in Appendix A.

Thus, the deflection function $w(x, y, t)$, stress function $\Psi(x, y, t)$ and electric potential function $\phi(x, y, t)$ in the nonlinear dynamic governing equations of GEP films under clamped boundary conditions are obtained.

Substituting Eqs. (23)-(25) into the nonlinear dynamic governing equation of GEP laminated films and utilizing the Galerkin method [42], the integral

$$
\int_{A} \Gamma(w, \Phi, \phi) \mathrm{X} d A
$$

can be calculated over the rectangle area of GEP laminated films, where $\Gamma(w, \Phi, \phi)$ is taken as the nonlinear governing equation (16) and $\mathrm{X}$ is the corresponding spatial part of deflection function (23). The nonlinear dynamic governing equation can be derived into undamped Helmholtz-Duffing's oscillator, as follows

$$
g_{0} \frac{d^{2} W(t)}{d t^{2}}+g_{1} W(t)+g_{2} W(t)^{2}+g_{3} W(t)^{3}=0
$$

where the coefficients in above equation are relation to the material behaviors, geometrical size, small scale effect and vibration modes of GEP laminated films, and are given in detail in Appendix B.

It is seen from Eq. (23) that the spatial part $\left(1-\cos \left(\alpha_{m} x\right)\right)\left(1-\cos \left(\beta_{n} y\right)\right)$ ranges 
from 0 to 4 resulting in the non-dimensional maximum amplitude $A=W_{m}^{*}=4 W_{m}$ so $W^{*}(t)=4 W(t)$ is adopted to replace $W(t)$ in Eq. (27) as

$$
g_{0}^{*} \frac{d^{2} W^{*}(t)}{d t^{2}}+g_{1}^{*} W^{*}(t)+g_{2}^{*} W^{*}(t)^{2}+g_{3}^{*} W^{*}(t)^{3}=0
$$

where

$$
g_{0}^{*}=g_{0}, \quad g_{1}^{*}=g_{1}, \quad g_{2}^{*}=\frac{g_{2}}{4}, \quad g_{3}^{*}=\frac{g_{3}}{16} .
$$

Utilizing the iterative homotopy harmonic balance method [43], the solution of Eq. (28) is given by

$$
\omega=\sqrt{\frac{g_{1}^{*}}{g_{0}^{*}}+\frac{8 g_{2}^{*}}{3 \pi g_{0}^{*}} A+\frac{3 g_{3}^{*}}{4 g_{0}^{*}} A^{2}}=\sqrt{\frac{g_{1}}{g_{0}}+\frac{2 g_{2}}{3 \pi g_{0}} A+\frac{3 g_{3}}{64 g_{0}} A^{2}} \text { or } f=\omega / 2 \pi
$$

Neglecting the terms $A$ and $A^{2}$ in Eq. (29) the linear frequency $\omega_{L}=\sqrt{g_{1} / g_{0}}$ of GEP laminated films is obtained without the impact of combined tension-bending.

\section{Results and discussions}

To validate the accuracy of present model, a comparison of resonant frequencies obtained by present model and molecular dynamics (MD) method [44] is demonstrated in Fig. 3, where the GEP laminated films reduces to a single-layered graphene when the thicknesses of elastic layer and piezoelectric layer are taken as zero. The dimensions of the graphene sheet are taken as the thickness $h_{g}=0.34 \mathrm{~nm}$, size length $L_{x}=10 \sim 50 \mathrm{~nm}$, and scale effect parameter $\mu=0.843 \mathrm{~nm}$, respectively. It is obvious that present results are exactly consistent with that of MD method in Ref. [44]. The further comparison with available results in the literature [38] is demonstrated by degenerating the nonlocal asymmetric GEP laminated films model in the paper into a nonlocal symmetric plate model, where the solution of nonlocal linear frequency under simply supported boundary conditions is the same as that in Ref. [38], as follows:

$$
\omega_{m n}=\sqrt{\frac{D_{11} \lambda_{m}^{4}+2\left(D_{12}+2 D_{66}\right) \lambda_{m}^{2} \lambda_{n}^{2}+D_{22} \lambda_{n}^{4}}{I_{0}\left[1+\mu^{2}\left(\lambda_{m}^{2}+\lambda_{n}^{2}\right)\right]}},
$$


where $\lambda_{m}=\frac{m \pi}{a}, \lambda_{n}=\frac{n \pi}{b}$, and the deducing steps are expressed in Appendix $\mathbf{C}$.

Following the above validation, several numerical examples are presented to discuss the influences of key factors including material properties, non-dimensional scale parameter $\zeta=e_{0} l / L_{x}$, mode numbers $(m, n)$, size length $a / h$ or $b / a$, non-dimensional vibration amplitude $w_{\max } / h$, and the strength of electric potential $V$ on the nonlinear resonant of GEP laminated films. All material properties used in examples are listed in Appendix D, where the piezoelectric layer is taken as PZT-4 material [45], and graphene layer, elastic layer and piezoelectric layer are bonded each other.

Due to the distinguished carbon solubility of different metal materials, Graphene can be fabricated on different metal foils [46] and transferred to other substrates by PMMA technology [47]. Also some new techniques have been devised to fabricate the graphene/elastic composite [48-49]. In view of this, the elastic layer is taken as some commonly used metal foils $(\mathrm{Cu}, \mathrm{Al}, \mathrm{Ti}, \mathrm{W})$ in the fabricating of single-layered graphene. In order to study the effect of elastic layer material and geometric nonlinearity, Fig. 4 depicts the curves of nonlinear/linear resonant frequencies ratio $f_{N L} / f_{L}$ versus non-dimensional amplitude $w_{\max } / h$ for different metal layer materials, where size length of GEP laminated films is taken as $a=b=200 \mathrm{~nm}$; the thickness of piezoelectric layer, elastic layer and graphene layer are taken as $h_{1}=5 \mathrm{~nm}, h_{2}=10 \mathrm{~nm}$ and $h_{3}=0.34 \mathrm{~nm}$, respectively; non-dimensional scale parameter $\zeta$ and electric potential $V$ are taken as 0.1 and 1.0 Volt, respectively. It is found that the nonlinear behavior of resonant frequencies nonlinearly increases with respect to $w_{\max } / h$, and appears the highest value for metal $\mathrm{W}$ owing to its largest elastic coefficients: $C_{i j}(\mathrm{~W})>$ $C_{i j}(\mathrm{Cu})>C_{i j}(\mathrm{Ti})>C_{i j}(\mathrm{Al})$. This trend denotes that the nonlinear resonant frequency of GEP laminated films has a close connection with the material properties of elastic layer. It is also found that the value of $f_{N L} / f_{L}$ approximately becomes as much as 1.9 for metal elastic material $\mathrm{W}$ when the amplitude of non-dimensional amplitude $w_{\max } / h$ reaches 2.0, manifesting that the large deflection significantly increase the nonlinear behavior of resonant frequency. 
In the active control system of GEP laminated films, the voltage exerted on piezoelectric layer plays an important role in the control of nonlinear resonant. Fig. 5 shows the curves of nonlinear/linear resonant frequency ratio $\left(f_{N L} / f_{L}\right)$ versus non-dimensional amplitude ratio $\left(w_{\max } / h\right)$ of GEP laminated films under different electric potentials $V$, where the non-dimensional scale parameter is taken as $\zeta=0.05$. It is seen from Fig. 5 that the influence of voltage exerted on piezoelectric layer on the ratio between nonlinear and linear resonant frequencies may be neglected when the non-dimensional amplitude ratio $w_{\max } / h$ of GEP laminated films is less than 0.3 ; when the non-dimensional amplitude ratio is larger than 0.3 the influence of applied voltage increases as the non-dimensional amplitude ratio increases; the applied positive voltage enhances the ratio between nonlinear and linear resonant frequencies of GEP laminated films, and the applied negative voltage induces the ratio between nonlinear and linear resonant frequencies to decrease. It is concluded that the influence of the applied positive voltage on the nonlinear resonant frequency is larger than that on the linear resonant frequency, and the influence of the applied negative positive voltage on the nonlinear resonant frequency is less than that on the linear resonant frequency.

Fig. 6 shows that the influence of active voltage on the nonlinear resonant frequency of GEP laminated films depends on the small scale effect, and the influence decreases as the small scale parameter increases. It is seen that the applied positive voltage induces the nonlinear resonant frequency to decreases as the voltage increases, and the applied negative voltage induces the nonlinear resonant frequency increases, so that the nonlinear resonant frequency of GEP laminated films can be controlled according to the engineering requirement for nano high frequency antenna and signal generator by adjusting voltage exerted on piezoelectric layer.

Fig. 7 plots the relations between nonlinear frequency behavior and non-dimensional amplitude $w_{\max } / h$ for different non-dimensional scale parameters $(\zeta=0.0-0.4)$. It is seen that the influence of non-dimensional scale parameters on the nonlinear resonant frequency of GEP laminated films increases as the non-dimensional amplitude ratio $w_{\max } / h$, the nonlinear resonant frequency predicted by nonlocal theory is lower than that from classical continuation theory, and when the 
non-dimensional vibration amplitude $w_{\max } / h$ is larger than 0.3 the nonlinear resonant frequency decreases as the small scale effect increases.

It is seen from Fig. 8 that the influence of small scale parameter on the nature frequency $(m=1, n=1)$ of GEP laminated films is much less than that on the high order frequency $(m=2, n=2)$, and the small scale effect induce the resonant frequency and the nonlinear behavior of resonant frequency to decrease.

Fig. 9 shows the variation of nonlinear resonant frequency with size length $a / h$ of GEP laminated films for different mode numbers $(m=1, n=1-4)$. It is obvious that the nonlinear resonant frequencies remarkably drop as the size $(a / h)$ of GEP laminated films increases, and the influence of size length on the nonlinear resonant frequencies for different vibration modes is identical.

It is seen in Fig. $\mathbf{1 0}$ that the influence of side length ratio $b / a$ on the resonant frequency of GEP laminated films considering different non-dimensional scale parameters, here the nonlinear resonant frequency of rectangle GEP laminated films for classical theory $(\zeta=0.0)$ is larger than that of nonlocal theory $(\zeta=0.05,0.1)$, and the influence of small scale parameter on the nonlinear resonant frequency of rectangle GEP laminated films decreases as the side length ratio increases. And it is evidence that the extension of GEP laminated films in the $y$ direction can dramatically reduce the nonlinear resonant frequency of the GEP laminated films when size length ratio (b/a) varies from 0.5 to 1.5 .

Graphene coating on original elastic/piezoelectric laminated films can not only make it show some extra excellent electric characteristics but also improves the stiffness characteristic of the original EP films resulting in the enhancement of nonlinear resonant frequency. As depicted in Fig. 11, the nonlinear resonant frequencies ratio $f_{N L, G E P} / f_{N L, E P}$ between two kinds of laminated films is about 1.27 , and gradually decreases to 1.2 as the non-dimensional amplitude increases to 1 , which indicates the graphene layer with thickness $0.34 \mathrm{~nm}$ can significantly increase the stiffness of EP (elastic/piezoelectric) laminated films. This means that graphene coating on laminated films may improves its functional behavior because of 
graphene's excellent electric and mechanical properties, especially for some nano-electric engineering in extreme circumstance.

Though many works have focused on the homogeneous and isotropic single-layered armchair graphene which is adopted in Fig. 3-Fig. 10, several literatures predicted the graphene as a form of anisotropic 2D material [50-51]. Thus, orthotropic graphene are considered to study the impact of elastic constants of graphene in Fig. 12. Results show that the nonlinear resonant frequency of GEP laminated films increases as the elastic constants ratio of graphene sheet increases, and the nonlinear vibration deflection of GEP laminated films sharply enhance the value of nonlinear frequency. It is concluded that the enhancement of stiffness of graphene significantly improves the fundamental frequency of GEP laminated films so that a better study on the dynamic characteristics of GEP laminated films should be promoted for the design of micro or nano-devises.

\section{Conclusions}

Considering a exciting and active control system, both nonlocal theory and von Karman type nonlinear strain-displacement relations are taken into account to analytically investigate the nonlinear resonant frequency of GEP laminated films. Some main conclusions are given by

(1) The nonlinear resonant frequency of GEP laminated films can be controlled according to engineering requirement for high frequency antenna, signal generator, and super-capacitors etc. by adjusting voltage exerted on piezoelectric layer.

(2) The material property of elastic layer, as well as geometry nonlinear, has a significant effect on the nonlinear resonant frequency of GEP laminated films, where the nonlinear behavior of resonant frequencies nonlinearly increases with respect to $w_{\max } / h$, and appears the highest value for metal $\mathrm{W}$ owing to its largest elastic coefficients.

(3) The nonlinear resonant frequency predicted by nonlocal theory is lower than that from classical continuation theory, and the nonlinear resonant frequency decreases as the small scale effect increases when the non-dimensional vibration amplitude 
$w_{\max } / h$ is larger than 0.3 .

(4) The influence of size length on the nonlinear resonant frequency for different vibration modes is identical, and the nonlinear resonant frequencies remarkably drop as the ratio of side length to thickness $(a / h)$ of GEP laminated films increases.

(5) Graphene sheet coating on EP laminated films increases its stiffness characteristic resulting in the enhancement of nonlinear vibration frequencies, so that the nonlinear resonant frequency $f_{N L, G E P}$ of GEP laminated films is much larger than that $f_{N L, E P}$ of EP laminated films.

\section{Acknowledgment}

This work was financial supported from National Science Foundation of China (NSFC) under Number 11172165.

\section{References}

[1] Novoselov KS, Geim AK, Morozov SV, Morozov SV, Jiang D, Zhang Y, Dubonos SV, Grigorieva IV, Firsov AA. Electric field effect in atomically thin carbon films. Science 2004;306:666-9.

[2] Novoselov KS, Geim AK, Morozov SV, Jiang D, Katsnelson MI, Grigorieva IV, Dubonos SV, Firsov AA. Two-dimensional gas of massless Dirac fermions in graphene. Nature 2005;438:197-200.

[3] Geim AK, Novoselov KS. The rise of graphene. Nat Mater 2007;6:183-91.

[4] Eda G, Fanchini G, Chhowalla M. Large-area ultrathin films of reduced graphene oxide as a transparent and flexible electronic material. Nat Nanotechnol $2008 ; 3: 270-4$.

[5] Geim AK. Graphene: status and prospects. Science 2009;324:1530-4.

[6] Qian Y, Wang C, Le ZG. Decorating graphene sheets with Pt nanoparticles using sodium citrate as reductant. Appl Surf Sci 2011;257:10758-62.

[7] Jomehzadeh E, Saidi AR. A study on large amplitude vibration of multilayered graphene sheets. Comp Mater Sci 2011;50:1043-51. 
[8] Behjat B, Khoshravan MR. Geometrically nonlinear static and free vibration analysis of functionally graded piezoelectric plates. Compos Struct 2012;94:874-82.

[9] Chandratre S, Sharm P. Coaxing graphene to be piezoelectric. Appl Phys Lett 2012;100:023114.

[10] Shin KY, Hong JY, Jang J. Flexible and transparent graphene films as acoustic actuator electrodes using inkjet printing. Chem Commun 2011;47:8527-9.

[11] YoungáLee K, ThanháTien N. Highly sensitive stretchable transparent piezoelectric nanogenerators. Energ Environ Sci 2013;6:169-75.

[12] Eswaraiah V, Balasubramaniam K, Ramaprabhu S. Functionalized graphene reinforced thermoplastic nanocomposites as strain sensors in structural health monitoring. J Mater Chem 2011;21:12626-8.

[13] Li X, Zhang G, Bai X, Sun X, Wang X, Wang E, Dai H. Highly conducting graphene sheets and Langmuir-Blodgett films. Nat Nanotechnol 2008;3:538-42.

[14] Xu SC, Man BY, Jiang SZ, Chen CS, Yang C, Liu M, Gao XG, Sun ZC, Zhang C. Flexible and transparent graphene-based loudspeakers. Appl Phys Lett 2013;102:151902.

[15] Bae S, Kim H, Lee Y, Xu X, Park JS, Zheng Y. Roll-to-roll production of 30-inch graphene films for transparent electrodes. Nat Nanotechnol 2010;5:574-8.

[16] Zeng Y, Zhao Y, Jiang Y. Investigate the interface structure and growth mechanism of high quality $\mathrm{ZnO}$ films grown on multilayer graphene layers. Appl Surf Sci 2014;301:391-5.

[17] Jie W, Hui YY, Zhang Y, Lau SP, Hao J. Effects of controllable biaxial strain on the Raman spectra of monolayer graphene prepared by chemical vapor deposition. Appl Phys Lett 2013;102:223112.

[18] Battista L, Mecozzi L, Coppola S, Vespini V, Grilli S, Ferraro P. Graphene and carbon black nano-composite polymer absorbers for a pyro-electric solar energy harvesting device based on LiNbO3 crystals. Appl Energ 2014;136:357-62.

[19] Lee CK. Theory of laminated piezoelectric plates for the design of distributed sensors/actuators. Part I: Governing equations and reciprocal relationships. The 
Journal of the Acoustical Society of America 1990;87:1144-58.

[20] Pai PF, Nayfeh AH, Oh K, Mook DT. A refined nonlinear model of composite plates with integrated piezoelectric actuators and sensors. Int J Solids Struct 1993;30:1603-30.

[21] Mitchell JA, Reddy JN. A refined hybrid plate theory for composite laminates with piezoelectric laminae. Int J Solids Struct 1995;32:2345-67.

[22] Liu GR, Dai KY, Lim KM. Static and vibration control of composite laminates integrated with piezoelectric sensors and actuators using the radial point interpolation method. Smart Mater Struct 2004;13:1438.

[23] Milazzo A, Orlando C. An equivalent single-layer approach for free vibration analysis of smart laminated thick composite plates. Smart Mater Struct 2012;21:075031.

[24] Phung-Van P, De Lorenzis L, Thai CH, Abdel-Wahab M, Abdel-Wahab M. Analysis of laminated composite plates integrated with piezoelectric sensors and actuators using higher-order shear deformation theory and isogeometric finite elements. Comp Mater Sci 2015;96:495-505.

[25] Kapuria S, Nath JK. Coupled global-local and zigzag-local laminate theories for dynamic analysis of piezoelectric laminated plates. J Sound Vib 2013; 332:306-25.

[26] Wankhade RL, Bajoria KM. Free vibration and stability analysis of piezolaminated plates using the finite element method. Smart Mater Struct 2013;22:125040.

[27] Farsangi MAA, Saidi AR. Levy type solution for free vibration analysis of functionally graded rectangular plates with piezoelectric layers. Smart Mater Struct 2012;21:094017.

[28] Sarangi SK, Ray MC. Active damping of geometrically nonlinear vibrations of laminated composite plates using vertically reinforced 1-3 piezoelectric composites. Acta Mech 2011;222:363-80.

[29] Suresh KR, Ray MC. Active control of geometrically nonlinear vibrations of doubly curved smart sandwich shells using 1-3 piezoelectric composites. 
Compos Struct 2013;105:173-87.

[30] Wong EW, Sheehan PE, Lieber CM. Nanobeam mechanics: elasticity, strength, and toughness of nanorods and nanotubes. Science 1997;277:1971-5.

[31] Yakobson BI, Brabec CJ, Bernholc J. Nanomechanics of carbon tubes: instability beyond linear response. Phys Rev Lett 1996;76:2511.

[32] Zhou LG., Shi SQ. Molecular dynamic simulations on tensile mechanical properties of single-walled carbon nanotubes with and without hydrogen storage. Comp Mater Sci 2002;23:166-74.

[33] Eringen AC. Linear theory of nonlocal elasticity and dispersion of plane waves. Int J Eng Sci 1972;10:425-35.

[34] Li C. A nonlocal analytical approach for torsion of cylindrical nanostructures and the existence of higher-order stress and geometric boundaries, Compos Struct 2014;118: 607 .

[35] Li C. Torsional vibration of carbon nanotubes: comparison of two nonlocal models and a semi-continuum model, Int J Mech Sci 2014;82: 25.

[36] Raghu P, Preethi K, Amirtham R, Reddy JN. Nonlocal third-order shear deformation theory for analysis of laminated plates considering surface stress effects. Compos Struct 2016;139:13-29.

[37] Preethi K, Amirtham R, Reddy JN. Surface and non-local effects for non-linear analysis of Timoshenko beams. Int J Nonlin Mech 2015;76:100-11.

[38] Lu P, Zhang PQ, Lee HP, Wang CM, Reddy JN. Non-local elastic plate theories, Proc R Soc A 2007; 463:3225-40.

[39] Nazemnezhad R, Hosseini-Hashemi S. Free vibration analysis of multi-layer graphene nanoribbons incorporating interlayer shear effect via molecular dynamics simulations and nonlocal elasticity. Phys Lett A 2014;378:3225-32.

[40] Asemi SR, Farajpour A, Mohammadi M. Nonlinear vibration analysis of piezoelectric nanoelectromechanical resonators based on nonlocal elasticity theory. Compos Struct 2014;116:703-12.

[41] Asemi SR, Farajpour A. Thermo-electro-mechanical vibration of coupled piezoelectric-nanoplate systems under non-uniform voltage distribution 
embedded in Pasternak elastic medium. Curr Appl Phys 2014;14:814-32.

[42] Jomehzadeh E, Saidi AR. A study on large amplitude vibration of multilayered graphene sheets. Comp Mater Sci 2011;50:1043-51.

[43] Guo Z, Leung AYT. The iterative homotopy harmonic balance method for conservative Helmholtz-Duffing oscillators. Appl Math Comput 2010;215:3163-9.

[44] Ansari R, Sahmani S, Arash B. Nonlocal plate model for free vibrations of single-layered graphene sheets. Phys Lett A 2010;375:53-62.

[45] Dong K, Wang X. Influence of large deformation and rotary inertia on wave propagation in piezoelectric cylindrically laminated shells in thermal environment. Int J Solids Struct 2006;43:1710-26.

[46] Cai W, Zhu Y, Li X, Piner RD, Ruoff RS. Large area few-layer graphene/graphite films as transparent thin conducting electrodes. Appl Phys Lett 2009;95:123115.

[47] Li X, Zhu Y, Cai W, Borysiak M, Han B, Chen D, Piner RD, Colombo R, Ruoff RS. Transfer of large-area graphene films for high-performance transparent conductive electrodes. Nano Lett 2009;9:4359-63.

[48] Rybkina AA, Rybkin AG, Fedorov AV, Usachov DYu, Yachmenev ME, Marchenko DE, Vilkov OYu, Nelyubov AV, Adamchuk VK, Shikin AM. Interaction of graphene with intercalated $\mathrm{Al}$ : The process of intercalation and specific features of the electronic structure of the system. Surf Sci 2013;609:7-17.

[49] Rybkina AA, Rybkin AG, Adamchuk VK, Marchenko D, Varykhalov A, Sánchez-Barriga J, Shikin AM. The graphene/Au/Ni interface and its application in the construction of a graphene spin filter. Nanotechnology 2013;24:295201.

[50] Shen L, Shen HS, Zhang CL. Nonlocal plate model for nonlinear vibration of single layer graphene sheets in thermal environments. Comp Mater Sci 2010; 48:680-5.

[51] Mortazavi B, Rajabpour A, Ahzi S, Rémond Y, Mehdi Vaez Allaei S. Nitrogen doping and curvature effects on thermal conductivity of graphene: A non-equilibrium molecular dynamics study. Solid State Commun 


\section{Appendix A:}

$$
\begin{aligned}
& A_{1}=-\frac{1}{2} \frac{\alpha_{m}^{2} \beta_{n}^{2}}{S_{11}\left(\left(2 \alpha_{m}\right)^{4}+\mu^{2}\left(2 \alpha_{m}\right)^{6}\right)}, A_{2}=-\frac{1}{2} \frac{\alpha_{m}^{2} \beta_{n}^{2}}{S_{22}\left(\left(2 \beta_{n}\right)^{4}+\mu^{2}\left(2 \beta_{n}\right)^{6}\right)}, A_{3}=\frac{1}{2} \frac{\alpha_{m}^{2} \beta_{n}^{2}}{\Theta_{1}}, \\
& A_{4}=\frac{1}{2} \frac{\alpha_{m}^{2} \beta_{n}^{2}}{\Theta_{2}}, A_{5}=-\frac{\alpha_{m}^{2} \beta_{n}^{2}}{\Theta_{3}}, A_{6}=\frac{1}{2} \frac{\alpha_{m}^{2} \beta_{n}^{2}}{S_{11}\left(\left(\alpha_{m}\right)^{4}+\mu^{2}\left(\alpha_{m}\right)^{6}\right)}, A_{7}=\frac{1}{2} \frac{\alpha_{m}^{2} \beta_{n}^{2}}{S_{22}\left(\left(\beta_{n}\right)^{4}+\mu^{2}\left(\beta_{n}\right)^{6}\right)}, \\
& B_{1}=-\frac{R_{12}\left(\alpha_{m}\right)^{4}+\left(R_{11}+R_{22}-2 R_{66}\right)\left(\alpha_{m} \beta_{n}\right)^{2}+R_{12}\left(\beta_{n}\right)^{4}}{\Theta_{3}}, \\
& B_{2}=-\frac{R_{12}\left(\alpha_{m}\right)^{4}}{S_{11}\left(\left(\alpha_{m}\right)^{4}+\mu^{2}\left(\alpha_{m}\right)^{6}\right)}, B_{3}=-\frac{R_{12}\left(\beta_{n}\right)^{4}}{S_{11}\left(\left(\beta_{n}\right)^{4}+\mu^{2}\left(\beta_{n}\right)^{6}\right)} .
\end{aligned}
$$

where

$$
\begin{aligned}
& \Theta_{1}=\left[S_{11}\left(2 \alpha_{m}\right)^{4}+2\left(S_{12}+2 S_{66}\right)\left(2 \alpha_{m} \beta_{n}\right)^{2}+S_{22}\left(\beta_{n}\right)^{4}\right]+\mu^{2}\left[S_{11}\left(2 \alpha_{m}\right)^{6}+\left(2\left(S_{12}+2 S_{66}\right)\right.\right. \\
& \left.\left.+S_{11}\right)\left(2 \alpha_{m}\right)^{4}\left(\beta_{n}\right)^{2}+\left(2\left(S_{12}+2 S_{66}\right)+S_{22}\right)\left(2 \alpha_{m}\right)^{2}\left(\beta_{n}\right)^{4}+S_{22}\left(\beta_{n}\right)^{6}\right] \\
& \Theta_{2}=\left[S_{11}\left(\alpha_{m}\right)^{4}+2\left(S_{12}+2 S_{66}\right)\left(\alpha_{m} \cdot 2 \beta_{n}\right)^{2}+S_{22}\left(2 \beta_{n}\right)^{4}\right]+\mu^{2}\left[S_{11}\left(\alpha_{m}\right)^{6}+\left(2\left(S_{12}+2 S_{66}\right)\right.\right. \\
& \left.\left.+S_{11}\right)\left(\alpha_{m}\right)^{4}\left(2 \beta_{n}\right)^{2}+\left(2\left(S_{12}+2 S_{66}\right)+S_{22}\right)\left(\alpha_{m}\right)^{2}\left(2 \beta_{n}\right)^{4}+S_{22}\left(2 \beta_{n}\right)^{6}\right] \\
& \Theta_{3}=\left[S_{11}\left(\alpha_{m}\right)^{4}+2\left(S_{12}+2 S_{66}\right)\left(\alpha_{m} \beta_{n}\right)^{2}+S_{22}\left(\beta_{n}\right)^{4}\right]+\mu^{2}\left[S_{11}\left(\alpha_{m}\right)^{6}+\left(2\left(S_{12}+2 S_{66}\right)\right.\right. \\
& \left.\left.+S_{11}\right)\left(\alpha_{m}\right)^{4}\left(\beta_{n}\right)^{2}+\left(2\left(S_{12}+2 S_{66}\right)+S_{22}\right)\left(\alpha_{m}\right)^{2}\left(\beta_{n}\right)^{4}+S_{22}\left(\beta_{n}\right)^{6}\right] \\
& C_{1}=\frac{3 \pi^{2}\left(S_{12} b^{2} m^{2}-S_{11} a^{2} n^{2}\right)}{2 a^{2} b^{2}\left(S_{12}^{2}-S_{11} S_{22}\right)}, C_{2}=\frac{3 \pi^{2}\left(S_{22} b^{2} m^{2}-S_{12} a^{2} n^{2}\right)}{2 a^{2} b^{2}\left(S_{11} S_{22}-S_{12}^{2}\right)}, C_{3}=0 .
\end{aligned}
$$

\section{Appendix B:}

$$
\begin{aligned}
g_{0} & =D_{9}^{*} \frac{3\left(3 a^{2} b^{2}+4 \mu^{2} \pi^{2}\left(a^{2} n^{2}+b^{2} m^{2}\right)\right)}{4 a b m n} \\
& -D_{10}^{*} \frac{\pi^{2}\left(3 a^{2} b^{2}\left(a^{2} n^{2}+b^{2} m^{2}\right)+4 \mu^{2} \pi^{2}\left(3 a^{4} n^{4}+2 a^{2} b^{2} m^{2} n^{2}+3 b^{4} m^{4}\right)\right)}{a^{3} b^{3} m n}
\end{aligned}
$$




$$
\begin{aligned}
g_{1}= & \frac{4 \pi^{4}\left(3 D_{1}^{*} b^{4} m^{4}+D_{2}^{*} a^{2} b^{2} m^{2} n^{2}+3 D_{3}^{*} a^{4} n^{4}\right)}{a^{3} b^{3} m n} \\
& -\frac{4 \pi^{4}\left(D_{6}^{*}\left(B_{1}-2 B_{3}\right) a^{4} n^{4}+D_{5}^{*} B_{1} a^{2} b^{2} m^{2} n^{2}+D_{4}^{*}\left(B_{1}-2 B_{2}\right) b^{4} m^{4}\right)}{a^{3} b^{3} m n} \\
& -\frac{16 \mu^{2} \pi^{6}\left(D_{6}^{*}\left(B_{1}-2 B_{3}\right) a^{6} n^{6}+B_{1} a^{2} b^{2} m^{2} n^{2}\left(\left(D_{5}^{*}+D_{6}^{*}\right) a^{2} n^{2}+\left(D_{5}^{*}+D_{4}^{*}\right) b^{2} m^{2}\right)+D_{4}^{*}\left(B_{1}-2 B_{2}\right) b^{6} m^{6}\right)}{a^{5} b^{5} m n}, \\
& +\frac{\pi^{2}\left(D_{7}^{*}\left(\varphi_{1}-2 \varphi_{2}\right) b^{2} m^{2}+D_{8}^{*}\left(\varphi_{1}-2 \varphi_{3}\right) a^{2} n^{2}\right)}{a b m n}-\frac{3 \pi^{2}\left(D_{11}^{*} b^{2} m^{2}+D_{12}^{*} a^{2} n^{2}\right)}{a b m n} \\
g_{2}= & -\frac{4 \pi^{4}\left(D_{4}^{*}\left(A_{5}-2 A_{6}\right) b^{4} m^{4}+D_{5}^{*} A_{5} a^{2} b^{2} m^{2} n^{2}+D_{6}^{*}\left(A_{5}-2 A_{7}\right) a^{4} n^{4}\right)}{a^{3} b^{3} m n} \\
& -\frac{16 \mu^{2} \pi^{6}\left(D_{6}^{*}\left(A_{5}-2 A_{7}\right) a^{6} n^{6}+A_{5} a^{2} b^{2} m^{2} n^{2}\left(\left(D_{5}^{*}+D_{6}^{*}\right) a^{2} n^{2}+\left(D_{5}^{*}+D_{4}^{*}\right) b^{2} m^{2}\right)+D_{4}^{*}\left(A_{5}-2 A_{6}\right) b^{6} m^{6}\right),}{a b} \\
& -\frac{8 \pi^{4}\left(B_{1}-B_{2}-B_{3}\right)}{a b m n} \\
g_{3}= & \frac{-4 \pi^{4} m n\left(2\left(A_{1}+A_{2}\right)-\left(A_{3}+A_{4}\right)+2\left(A_{5}-A_{6}-A_{7}\right)\right)}{a b}+\frac{3 \pi^{2}\left(C_{1} a^{2} n^{2}+C_{2} b^{2} m^{2}\right)}{a b m n} .
\end{aligned}
$$

\section{Appendix C:}

The main deducing process for linear frequency of a nonlocal symmetric thin plate model:

Eq. (16) could be degenerated into

$$
D_{11} \frac{\partial^{4} w}{\partial x^{4}}+2\left(D_{12}+2 D_{66}\right) \frac{\partial^{4} w}{\partial x^{2} \partial y^{2}}+D_{22} \frac{\partial^{4} w}{\partial y^{4}}+\left(1-\mu^{2} \nabla^{2}\right) I_{0} \frac{\partial^{2} w}{\partial t^{2}}=0
$$

The deflection function that satisfies the simply supported boundary conditions can be expressed as following

$$
w(x, y, t)=W(t) \sin \left(\lambda_{m} x\right) \sin \left(\lambda_{n} y\right),
$$

Then substituting Eq. (C-2) into Eq. (C-1) and using the Galerkin method, it can be derived as

$$
I_{0}\left[1+\mu^{2}\left(\lambda_{m}^{2}+\lambda_{n}^{2}\right)\right] \ddot{W}(t)+\left[D_{11} \lambda_{m}^{4}+2\left(D_{12}+2 D_{66}\right) \lambda_{m}^{2} \lambda_{n}^{2}+D_{22} \lambda_{n}^{4}\right] W(t)=0
$$

And the solution can be obtained as

$$
\omega_{m n}=\sqrt{\frac{D_{11} \lambda_{m}^{4}+2\left(D_{12}+2 D_{66}\right) \lambda_{m}^{2} \lambda_{n}^{2}+D_{22} \lambda_{n}^{4}}{I_{0}\left[1+\mu^{2}\left(\lambda_{m}^{2}+\lambda_{n}^{2}\right)\right]}}
$$




\section{Appendix D:}

The material properties of graphene:

Elastic constants: $E_{1}=E_{2}=1.0 \mathrm{TPa}, v=0.16, \rho=2250 \mathrm{Kg} / \mathrm{m}^{3}, h=0.34 \mathrm{~nm}$. Stiffness coefficients: $C_{11}=1.0263 \mathrm{TPa}, C_{12}=0.1642 \mathrm{TPa}, C_{22}=1.0263 \mathrm{TPa}, C_{66}=0.43103 \mathrm{TPa}$. The material properties of elastic layer:

Table. 1. Material stiffness coefficients of several elastic layers

\begin{tabular}{ccccc}
\hline \multirow{2}{*}{ Metal type } & \multicolumn{4}{c}{ stiffness coefficient (GPa) } \\
\cline { 2 - 5 } & $C_{11}$ & $C_{12}$ & $C_{22}$ & $C_{66}$ \\
\hline $\mathrm{Al}$ & 105 & 51 & 105 & 51 \\
$\mathrm{Cu}$ & 133 & 43 & 133 & 45 \\
$\mathrm{~W}$ & 374 & 104 & 374 & 130 \\
$\mathrm{Ti}$ & 112 & 33 & 112 & 38 \\
\hline
\end{tabular}

The material properties of piezoelectric material PZT-4:

Stiffness coefficients: $C_{11}=132 \mathrm{GPa}, C_{12}=71 \mathrm{GPa}, C_{22}=132 \mathrm{GPa}, C_{66}=26 \mathrm{GPa}$; Piezoelectric constants: $e_{31}=-4.1 C / \mathrm{m}^{2}, e_{32}=-4.1 \mathrm{C} / \mathrm{m}^{2}, e_{33}=14.1 \mathrm{C} / \mathrm{m}^{2}, e_{15}=10.5 \mathrm{C} / \mathrm{m}^{2}$; Dielectric constants: $g_{11}=5.841 \times 10^{-9} \mathrm{C} / \mathrm{V} \cdot \mathrm{m}, g_{33}=5.841 \times 10^{-9} \mathrm{C} / \mathrm{V} \cdot \mathrm{m}$.

\section{Caption of Figures}

Fig. 1. The schematic of a GEP (graphene/elastic/piezoelectric) asymmetric laminated films with a feedback control system.

Fig. 2. The coordinate of a rectangular GEP asymmetric laminated films in the $z$ direction.

Fig. 3. A compare of linear resonant frequency for clamped single-layered armchair graphene sheet obtained by different methods ( $a=b=10 \mathrm{~nm}, \mu^{2}=0.71 \mathrm{~nm}^{2}, \mathrm{E}=1.0 \mathrm{TPa}, v=0.16, h=0.34 \mathrm{~nm}$ ).

Fig.4. The ratio of nonlinear to linear resonant frequency for several metal elastic layers $(\mathrm{Al}, \mathrm{Cu}$, $\mathrm{W}, \mathrm{Ti}$ ), where size length $\mathrm{a}=\mathrm{b}=200 \mathrm{~nm}$; thickness of piezoelectric layer $h_{1}=5 \mathrm{~nm}$, elastic layer $h_{2}=10 \mathrm{~nm}$, graphene layer $h_{3}=0.34 \mathrm{~nm}$; scale parameter $\zeta=0.1$; active voltage $V=1.0$ Volt.

Fig.5. Nonlinear/linear resonant frequency ratio under different active $V$, where size length $a=b=200 \mathrm{~nm}$; thickness of piezoelectric layer $h_{1}=5 \mathrm{~nm}$, elastic layer $h_{2}=10 \mathrm{~nm}$, graphene layer $h_{3}=0.34 \mathrm{~nm}$; scale parameter $\zeta=0.05$.

Fig.6. Nonlinear resonant frequency versus active voltage $V$ for different scale parameter, where 
thickness of piezoelectric layer $h_{1}=5 \mathrm{~nm}$, elastic layer $h_{2}=10 \mathrm{~nm}$, graphene layer $h_{3}=0.34 \mathrm{~nm}$; size length $a=b=200 \mathrm{~nm}$; non-dimensional amplitude $w_{\max } / h=1.0$.

Fig.7. Nonlinear/linear resonant frequency ratio for different scale parameter $\zeta$, where size length $a=b=200 \mathrm{~nm}$; thickness of piezoelectric layer $h_{1}=5 \mathrm{~nm}$, elastic layer $h_{2}=10 \mathrm{~nm}$, graphene layer $h_{3}=0.34 \mathrm{~nm}$; active voltage $V=1.0$ Volt.

Fig.8. Resonant frequency versus scale parameter $\zeta$ for different mode number $(m, n)$, where size length $a=b=200 \mathrm{~nm}$; thickness of piezoelectric layer $h_{1}=5 \mathrm{~nm}$, elastic layer $h_{2}=10 \mathrm{~nm}$, graphene layer $h_{3}=0.34 \mathrm{~nm}$; active voltage $V=1.0$ Volt; non-dimensional amplitude $w_{\max } / h=1.0$.

Fig.9. Nonlinear resonant frequency versus size length $(a / h)$ for different mode number $(m, n)$, where thickness of piezoelectric layer $h_{1}=5 \mathrm{~nm}$, elastic layer $h_{2}=10 \mathrm{~nm}$, graphene layer $h_{3}=0.34$ $\mathrm{nm}$; active voltage $V=1.0$ Volt; non-dimensional amplitude $w_{\max } / h=1.0$.

Fig.10. Nonlinear vibration frequency for different scale parameter $\zeta$ versus size length ratio $(b / a)$, where size length $a=200 \mathrm{~nm}$; thickness of piezoelectric layer $h_{1}=5 \mathrm{~nm}$, elastic layer $h_{2}=10 \mathrm{~nm}$, graphene layer $h_{3}=0.34 \mathrm{~nm}$; active voltage $V=1.0$ Volt; non-dimensional amplitude $w_{\max } / h=1.0$.

Fig.11. Nonlinear resonant frequency ratio for GEP laminated films and EP (without graphene layer) laminated films, where size length $a=b=200 \mathrm{~nm}$; thickness of piezoelectric layer $h_{1}=5 \mathrm{~nm}$, elastic layer $h_{2}=10 \mathrm{~nm}$, graphene layer $h_{3}=0.34 \mathrm{~nm}$; active voltage $V=1.0$ Volt.

Fig.12. Nonlinear resonant frequency versus elastic constants ratio for different non-dimensional amplitude, where size length $a=b=200 \mathrm{~nm}$; thickness of piezoelectric layer $h_{1}=5 \mathrm{~nm}$, elastic layer $h_{2}=10 \mathrm{~nm}$, graphene layer $h_{3}=0.34 \mathrm{~nm}$; scale parameter $\zeta=0.1$; active voltage $V=1.0$ Volt. 


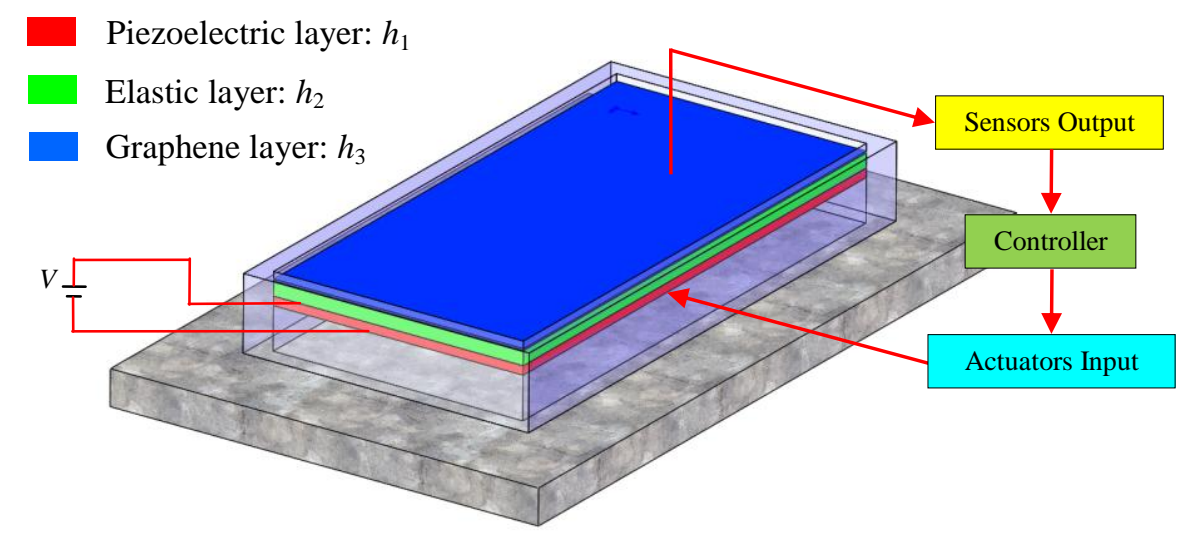

Fig. 1. The schematic of a GEP (graphene/elastic/piezoelectric) asymmetric laminated films with a feedback control system.

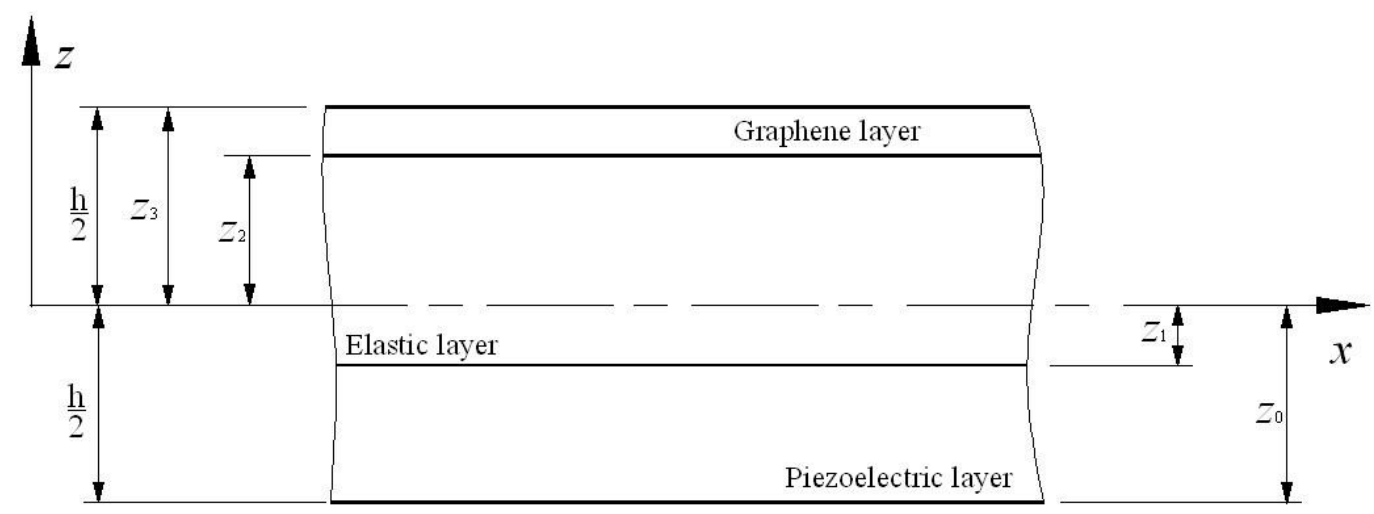

Fig. 2. The coordinate of a rectangular GEP asymmetric laminated films in the $z$ direction. 


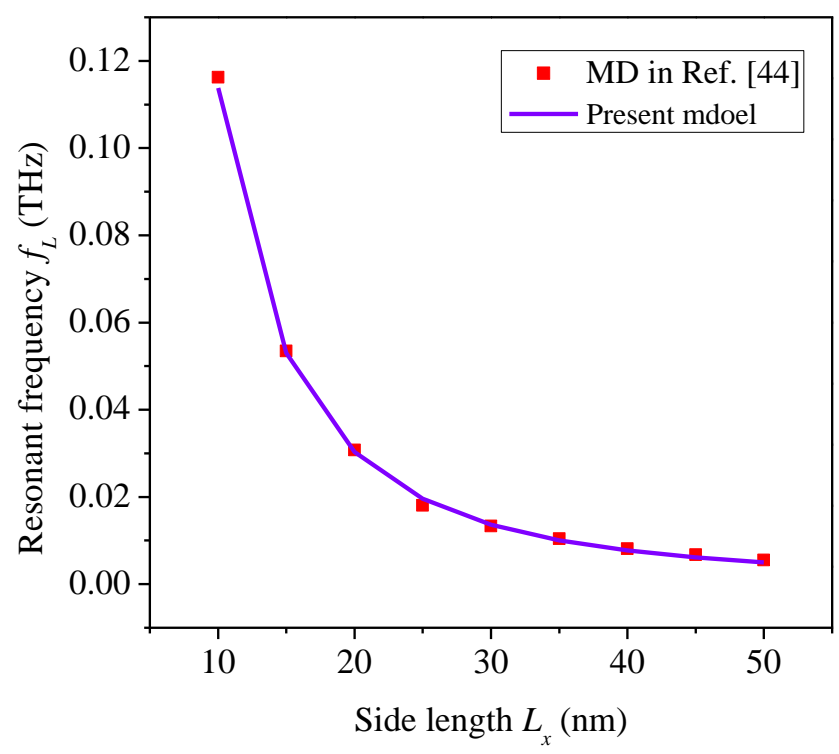

Fig. 3. A compare of linear resonant frequency for clamped single-layered armchair graphene sheet obtained by different methods ( $a=b=10 \mathrm{~nm}, \mu^{2}=0.71 \mathrm{~nm}^{2}, \mathrm{E}=1.0 \mathrm{TPa}, \nu=0.16, h=0.34 \mathrm{~nm}$ ).

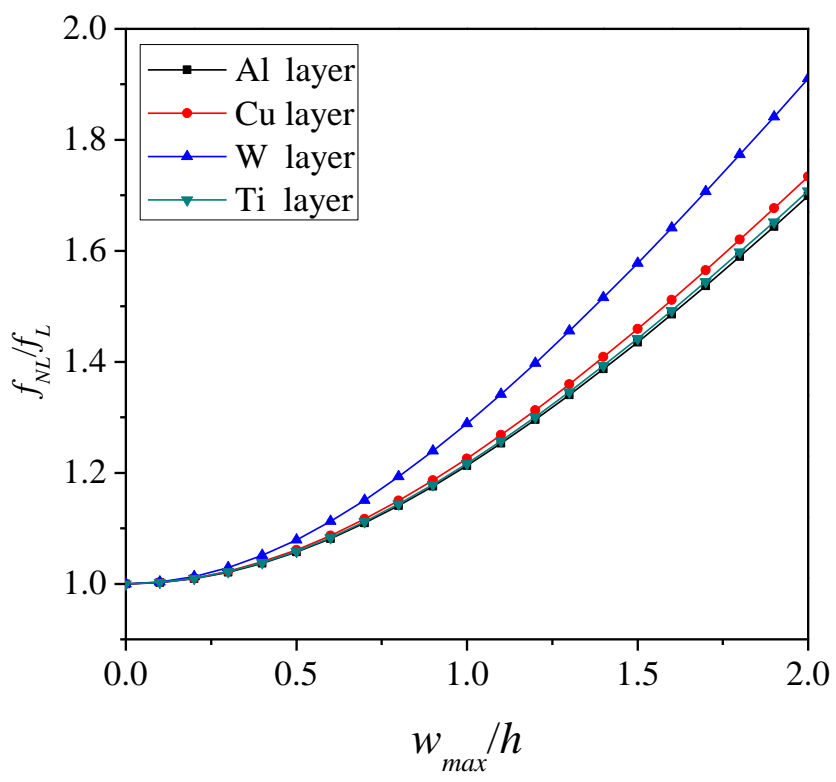

Fig.4. The ratio of nonlinear to linear resonant frequency for several metal elastic layers $(\mathrm{Al}, \mathrm{Cu}$, $\mathrm{W}, \mathrm{Ti}$ ), where size length $\mathrm{a}=\mathrm{b}=200 \mathrm{~nm}$; thickness of piezoelectric layer $h_{1}=5 \mathrm{~nm}$, elastic layer $h_{2}=10 \mathrm{~nm}$, graphene layer $h_{3}=0.34 \mathrm{~nm}$; scale parameter $\zeta=0.1$; active voltage $V=1.0$ Volt. 


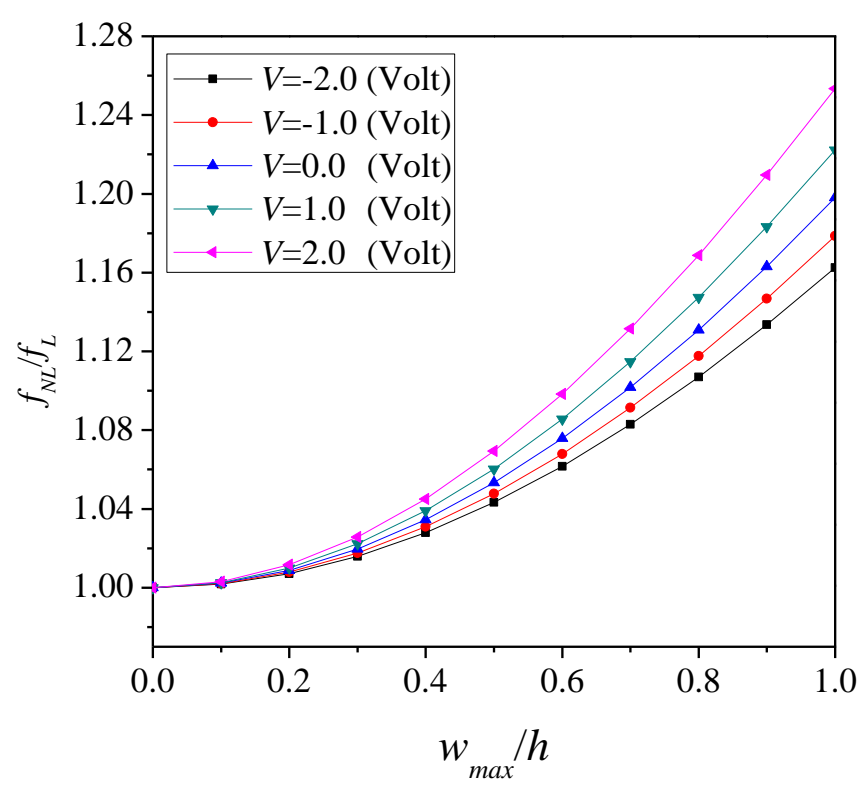

Fig.5. Nonlinear/linear resonant frequency ratio under different active voltages $V$, where size length $a=b=200 \mathrm{~nm}$; thickness of piezoelectric layer $h_{1}=5 \mathrm{~nm}$, elastic layer $h_{2}=10 \mathrm{~nm}$, graphene layer $h_{3}=0.34 \mathrm{~nm}$; scale parameter $\zeta=0.05$.

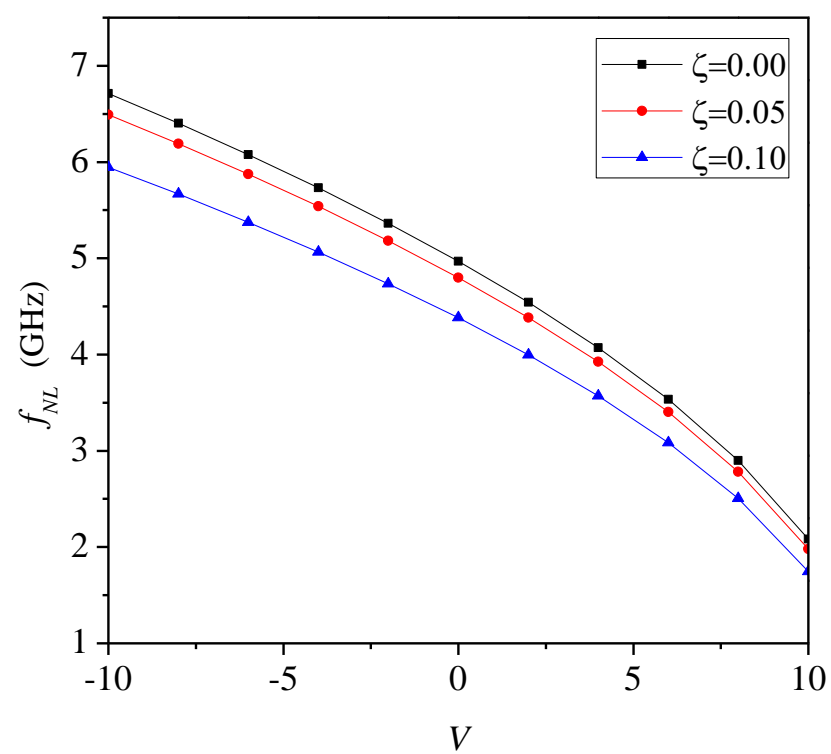

Fig. 6. Nonlinear resonant frequency versus active voltage $V$ for different scale parameter, where thickness of piezoelectric layer $h_{1}=5 \mathrm{~nm}$, elastic layer $h_{2}=10 \mathrm{~nm}$, graphene layer $h_{3}=0.34 \mathrm{~nm}$; size length $a=b=200 \mathrm{~nm}$; non-dimensional amplitude $w_{\max } / h=1.0$. 


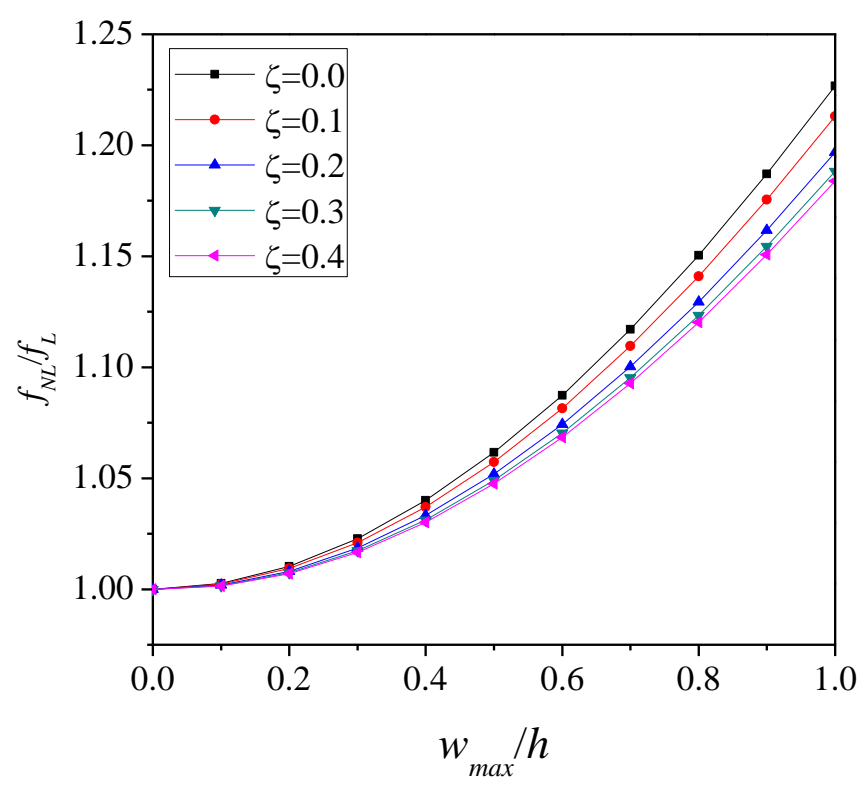

Fig.7. Nonlinear/linear resonant frequency ratio for different scale parameter $\zeta$, where size length $a=b=200 \mathrm{~nm}$; thickness of piezoelectric layer $h_{1}=5 \mathrm{~nm}$, elastic layer $h_{2}=10 \mathrm{~nm}$, graphene layer $h_{3}=0.34 \mathrm{~nm}$; active voltage $V=1.0$ Volt.

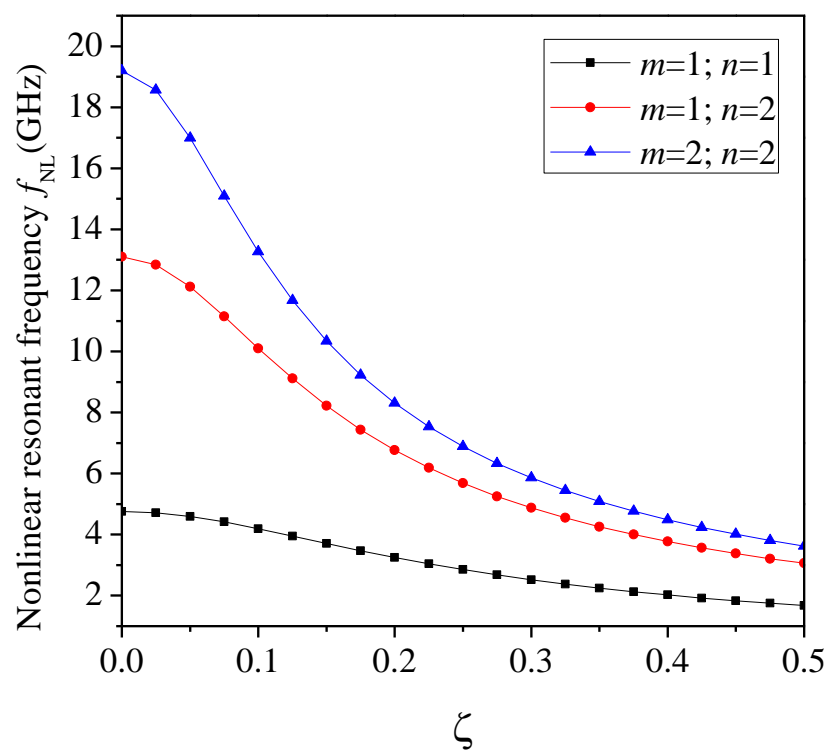

Fig.8. Resonant frequency versus scale parameter $\zeta$ for different mode number $(m, n)$, where size length $a=b=200 \mathrm{~nm}$; thickness of piezoelectric layer $h_{1}=5 \mathrm{~nm}$, elastic layer $h_{2}=10 \mathrm{~nm}$, graphene layer $h_{3}=0.34 \mathrm{~nm}$; active voltage $V=1.0$ Volt; non-dimensional amplitude $w_{\max } / h=1.0$. 


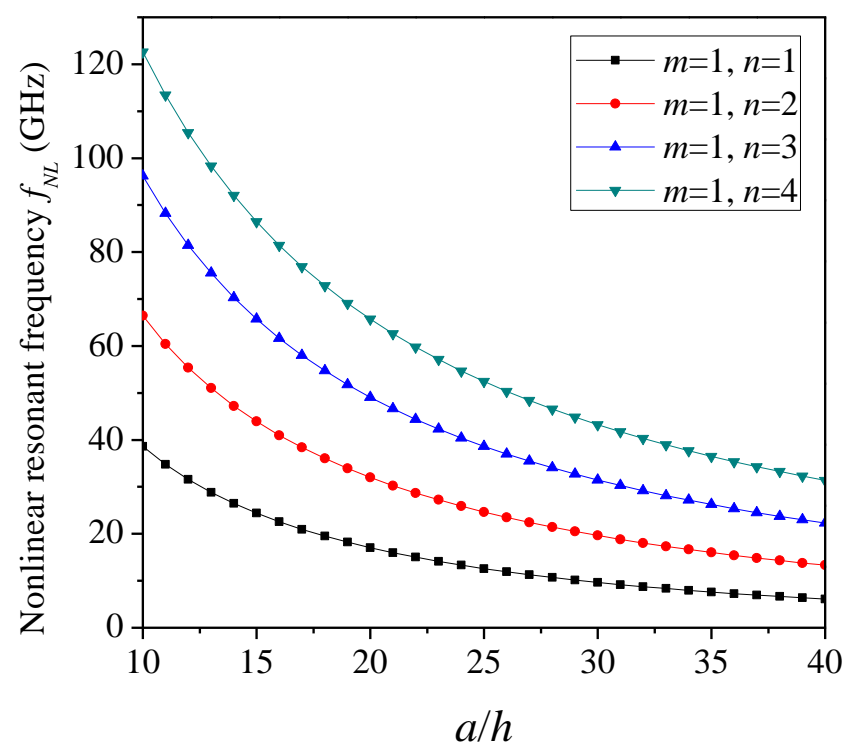

Fig.9. Nonlinear resonant frequency versus size length $(a / h)$ for different mode number $(m, n)$, where thickness of piezoelectric layer $h_{1}=5 \mathrm{~nm}$, elastic layer $h_{2}=10 \mathrm{~nm}$, graphene layer $h_{3}=0.34$ $\mathrm{nm}$; active voltage $V=1.0$ Volt; non-dimensional amplitude $w_{\max } / h=1.0$.

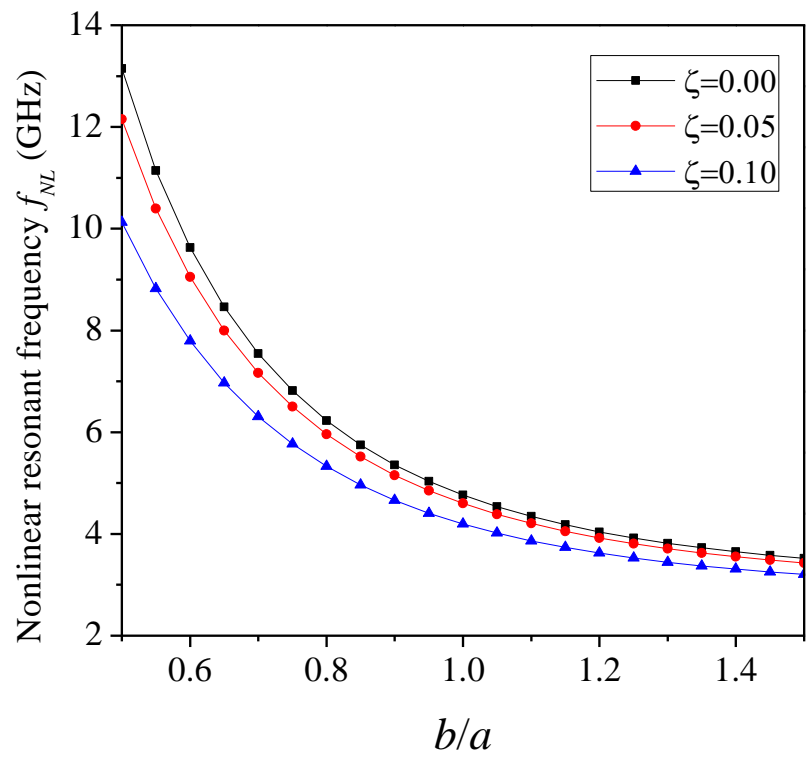

Fig.10. Nonlinear vibration frequency for different scale parameter $\zeta$ versus size length ratio $(b / a)$, where size length $a=200 \mathrm{~nm}$; thickness of piezoelectric layer $h_{1}=5 \mathrm{~nm}$, elastic layer $h_{2}=10 \mathrm{~nm}$, graphene layer $h_{3}=0.34 \mathrm{~nm}$; active voltage $V=1.0$ Volt; non-dimensional amplitude $w_{\max } / h=1.0$. 


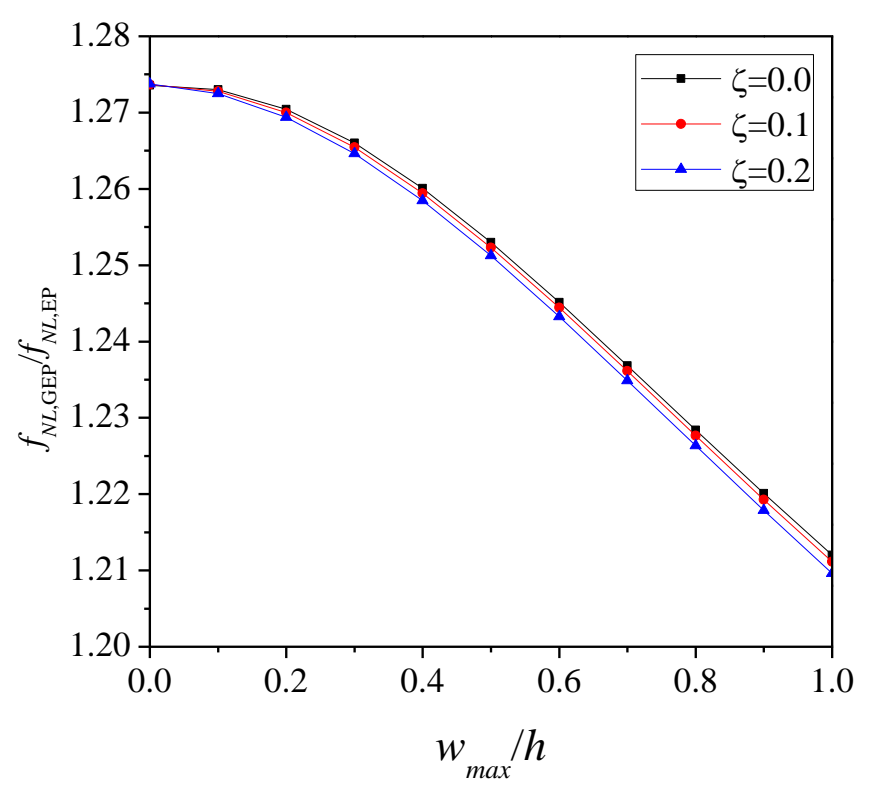

Fig.11. Nonlinear resonant frequency ratio for GEP laminated films and EP (without graphene layer) laminated films, where size length $a=b=200 \mathrm{~nm}$; thickness of piezoelectric layer $h_{1}=5 \mathrm{~nm}$, elastic layer $h_{2}=10 \mathrm{~nm}$, graphene layer $h_{3}=0.34 \mathrm{~nm}$; active voltage $V=1.0$ Volt.

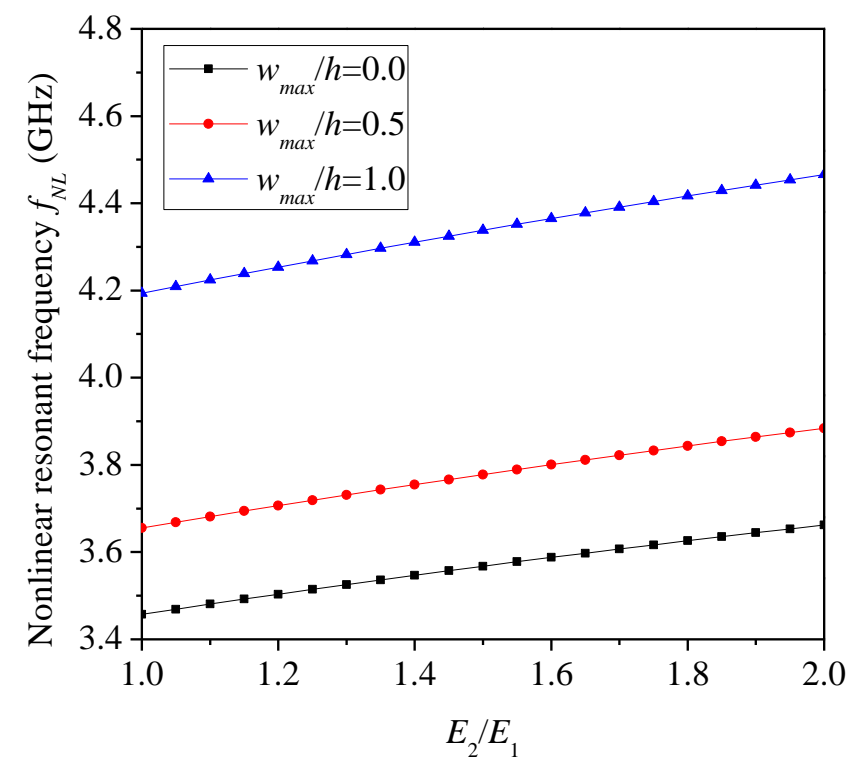

Fig.12. Nonlinear resonant frequency versus elastic constants ratio for different non-dimensional amplitude, where size length $a=b=200 \mathrm{~nm}$; thickness of piezoelectric layer $h_{1}=5 \mathrm{~nm}$, elastic layer $h_{2}=10 \mathrm{~nm}$, graphene layer $h_{3}=0.34 \mathrm{~nm}$; scale parameter $\zeta=0.1$; active voltage $V=1.0$ Volt. 


\section{Graphical abstract}

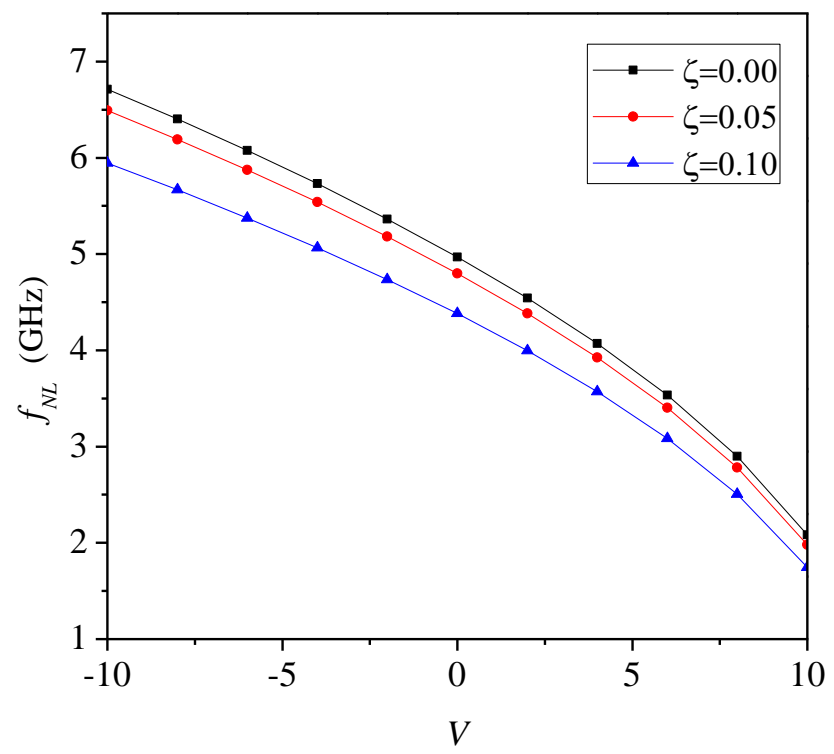

Fig. 1. Nonlinear resonant frequency versus active voltage $V$ for different scale parameter, where thickness of piezoelectric layer $h_{1}=5 \mathrm{~nm}$, elastic layer $h_{2}=10 \mathrm{~nm}$, graphene layer $h_{3}=0.34 \mathrm{~nm}$; size length $a=b=200 \mathrm{~nm}$; non-dimensional amplitude $w_{\max } / h=1.0$. 